\title{
Modeling Adsorption in Metal-Organic Frameworks with Open Metal Sites: Propane/Propylene Separations
}

\author{
Michael Fischer ${ }^{1,2}$, José R. B. Gomes ${ }^{3}$, Michael Fröba $^{1 *}$ and Miguel Jorge ${ }^{4 *}$ \\ ${ }^{1}$ Institute of Inorganic and Applied Chemistry, University of Hamburg, Martin-Luther-King-Platz 6, \\ 20146 Hamburg, Germany \\ Email-froeba@chemie.uni-hamburg.de \\ ${ }^{2}$ Department of Chemistry, University College London, 20 Gordon Street, London WC1H 0AJ, United \\ Kingdom \\ ${ }^{3}$ CICECO - Center for Research in Ceramics and Composite Materials, Department of Chemistry, \\ University of Aveiro, Campus Universitário de Santiago, 3810-193 Aveiro, Portugal \\ ${ }^{4}$ LSRE - Laboratory of Separation and Reaction Engineering - Associate Laboratory LSRE/LCM, \\ Faculdade de Engenharia, Universidade do Porto, Rua Dr. Roberto Frias, 4200-465 Porto, Portugal \\ Email-mjorge@fe.up.pt
}

\begin{abstract}
In this paper we present a new approach for modeling adsorption in Metal-Organic Frameworks (MOFs) with unsaturated metal centers, and apply it to the challenging propane/propylene separation in copper (II) benzene-1,3,5-tricarboxylate (CuBTC). We obtain information about the specific interactions between olefins and the open metal sites of the MOF using quantum mechanical density functional theory. A proper consideration of all the relevant contributions to the adsorption energy enables us to extract the component that is due to specific attractive interactions between the $\pi$ orbitals of the alkene and the coordinatively unsaturated metal. This component is fitted using a combination of a Morse potential and a power law function, and is then included into classical grand canonical Monte Carlo simulations of adsorption. Using this modified potential, together with a standard Lennard-Jones model, we are able to predict the adsorption of both propane (where no specific interactions are present) and propylene (where specific interactions are dominant). Binary adsorption isotherms for this mixture are in reasonable agreement with Ideal Adsorbed Solution Theory predictions. We compare our approach with previous attempts to predict adsorption in MOFs with open metal sites, and suggest possible future routes for improving our model.
\end{abstract}




\section{1 - Introduction}

Metal-organic frameworks (MOFs), also known as porous coordination polymers, have attracted a great deal of attention in the scientific community over recent years, due to their potential for adsorptive separations [1,2], gas storage [3,4], catalysis [5,6] and chemical sensing [7,8], among a variety of other possible applications [9]. Their interest as adsorbents, both from a fundamental and a practical standpoint, stems mostly from three properties that are, to a certain extent, specific to MOFs: i) unusually large pore volumes and surface areas, compared to more conventional adsorbents [10]; ii) framework flexibility [11]; iii) coordinatively unsaturated metal sites (also called "open" metal sites) [12]. Open metal sites (OMS), in particular, have been shown to have a decisive effect on the ability of MOFs to preferentially adsorb certain components from a gas mixture, thus remarkably increasing their adsorption selectivity [13]. Apart from those unusual properties, the nature of MOFs and of their synthesis process potentially allows for a priori material design, since one can combine different inorganic centers and organic linkers to obtain solids with specific structural and chemical properties [14]. To really achieve such a goal, however, it is first necessary to establish consistent links between a material's properties and its performance as an adsorbent - in other words, to be able to accurately predict adsorption in MOFs.

Molecular simulation has been used as an adsorption prediction tool in nanoporous materials for decades, both in crystalline frameworks, such as zeolites [15], and in more disordered solids, like activated carbons [16] or mesoporous silica [17], and has more recently been applied to MOFs with some success [18]. However, as highlighted recently [19], the extension of conventional simulation methods and molecular models to MOFs is not always straightforward, and sometimes results in dramatic failures. From the three specific characteristics of MOFs that were identified in the previous paragraph, the first one is arguably the easiest to tackle from a modeling perspective - since MOFs are crystalline materials, as long as a correct description of the crystallographic structure is available, the correct pore volume and surface area of the material should in principle arise directly from the model. Nevertheless, it is important to point out that discrepancies are sometimes observed between simulation and experiment, most likely due to the fact that the model considers a perfect crystal structure, while the real material may contain impurities and/or defects [19]. Framework flexibility is significantly more difficult to model, but several encouraging steps have already been taken to incorporate flexibility into adsorption theories [20] and to develop "flexible" molecular models for MOFs [21].

As for OMS, it has been clearly demonstrated that conventional force fields are inadequate for describing the specific interactions between the unsaturated metal and certain adsorbates [22-26], but only very recently have theoreticians attempted to address this problem. After recognizing that conventional models, while performing well for alkane adsorption, were unable to predict adsorption of alkenes in CuBTC (copper (II) benzene-1,3,5-tricarboxylate (btc) MOF with structural formula $\mathrm{Cu}_{3}(\mathrm{btc})_{2}$ containing OMS), Jorge and co-workers proposed a simplified model that relied on empirically adjusting the Lennard-Jones (LJ) parameter for the energy of interaction between the metal and the $\mathrm{sp}^{2}$ carbon atoms $[22,23]$. Pure component isotherms obtained with the improved model showed reasonable agreement with experiment [22], but it was later shown that selectivities from propane/propylene mixtures were significantly underestimated [23]. Fischer et al. [24] also tuned the 
LJ parameters, now for acetylene and carbon dioxide in CuBTC (both the energy and the collision radius simultaneously), but used a theoretically more consistent approach - they adjusted the parameters until the interaction energy profiles as a function of distance to the metal atom for the model closely matched results of quantum mechanical density functional theory (DFT) in small representative atomic clusters. Significant improvements were observed in the description of purecomponent adsorption isotherms of those two gases. Later, the same group performed a similar analysis for hydrogen adsorption in a series of MOFs with unsaturated $\mathrm{Cu}$ sites (including $\mathrm{CuBTC}$ ), but used a Morse potential for the $\mathrm{H}_{2}-\mathrm{Cu}$ interactions, since it resulted in a better fit of the corresponding DFT curves [25]. Not only did the new model provide much better agreement with experimental isotherms than the conventional force fields, but also showed a reasonable degree of transferability between structurally distinct $\mathrm{Cu}$-containing MOFs. Finally, a recent paper by Chen et al. [26] also used a combination of quantum mechanics and classical molecular simulations, but instead of adjusting the potential parameters, they mapped the interaction energies between methane and $\mathrm{CuBTC}$ calculated by dispersion-corrected DFT onto a regular three-dimensional grid, and used the grid directly to compute potential energies in the classical simulations. Once again, significant improvements in the description of methane adsorption isotherms were achieved by including the results of quantum calculations. This approach has the advantage of circumventing the limitations of classical force fields, but unfortunately it is not clear how it can be extended to more complex, nonspherically symmetric, adsorbates.

All studies described above agree on the fact that a correct description of the specific interactions between certain adsorbates and OMS is crucial to obtain accurate predictions of adsorption isotherms, and that a combination of quantum mechanical calculations and classical molecular simulations has the potential to solve the problem. In this paper, we improve the approach of Fischer et al. $[24,25]$ by considering larger molecular clusters, a more accurate DFT approach and a more realistic consideration of the different contributions to the adsorption energy, and apply it to describe propane/propylene separations in CuBTC. The separation of propane from propylene is among the most difficult and energy intensive operations in industry, and tremendous cost and energy savings are expected if one is able to design an adsorbent with improved properties for this separation [27]. Recently, MOFs with OMS have shown great potential for separating these challenging mixtures [28,29]. CuBTC [30], also known as HKUST-1, was chosen because it is one of the most widely studied MOFs, it is an archetypal example of a MOF with OMS, and because recent experimental data for propane/propylene adsorption are available on that MOF [22]. We systematically investigate the effect of the size of the molecular cluster, of the degrees of freedom in the DFT optimization, and, most importantly, of the strategy for incorporating the DFT energies into the classical simulations, on the resulting predictions of propane and propylene adsorption in CuBTC. A consistent and generally applicable methodology for modeling adsorption in MOFs with OMS is proposed, its performance is tested by predicting binary propane/propylene adsorption in CuBTC, and possible routes for further improvement are discussed. 


\section{2 - Computational details}

\subsection{Grand Canonical Monte Carlo simulations}

All equilibrium adsorption isotherms were calculated using the grand canonical Monte Carlo (GCMC) method [31], in which the temperature $(T)$, volume $(V)$ and chemical potential $(\mu)$ of the system are kept fixed, while the total number of molecules $(N)$ is allowed to fluctuate. MC trials consisted of random translation + rotation $(40 \%)$, using a maximum displacement of $0.2 \mathrm{~nm}$, creation of a new adsorbate molecule at a random position in the simulation cell $(30 \%)$ and deletion of a random adsorbate molecule (30\%). For each pure-component isotherm point, the system was equilibrated during 5,000,000 steps, followed by 20,000,000 sampling steps for data collection. Mixture calculations used 20,000,000 steps for equilibration and 50,000,000 steps for sampling. The sampling stage was divided into 20 equal-length blocks for statistical averaging purposes. The chemical potential was converted to pressure $(P)$ by applying the Peng-Robinson equation of state [32]. In order to compare the simulated isotherms to experimental data, the former must be converted from absolute to excess adsorbed amount. We follow the approach of Myers and Monson [33], and calculate the difference between absolute and excess adsorption from the second virial coefficient for helium adsorption. An in-house computer code was used for all the isotherm calculations because it allowed for straightforward inclusion of the modified potential energy functional forms used to represent specific interactions with the OMS of the framework, as described in section 2.3.

The CuBTC framework was modeled by a rigid all-atom representation of a single unit cell, using the atomic positions from the experimental crystal structure [30], which is depicted in Figure 1. This MOF, with structural formula $\mathrm{Cu}_{3}(\mathrm{btc})_{2}$ ( $\mathrm{btc}=1,3,5$-benzene tricarboxylate), is composed of copper dimers coordinated to the oxygen atoms of the btc linkers. The key structural characteristic is a copper paddlewheel with a $\mathrm{Cu}-\mathrm{Cu}$ distance of $2.63 \AA$ A. Each copper atom is coordinated to another $\mathrm{Cu}$ atom, four oxygen atoms from the btc linkers and one water (solvent) molecule. Prior to experimental measurements of adsorption, these water molecules are removed by an activation procedure (usually heating under vacuum), leaving unsaturated $\mathrm{Cu}$ sites available for adsorption. Therefore, in this work the dehydrated form of $\mathrm{CuBTC}$ was considered in all adsorption simulations. Lennard-Jones potential parameters for the framework were taken from the DREIDING force field [34], except those for the $\mathrm{Cu}$ atom, which were not available in DREIDING and were thus taken from UFF [35]. The validity of these parameters for describing adsorption of hydrocarbons in IRMOF-1 and IRMOF-6, which are built from the same type of organic ligand as CuBTC but contain no OMS, has been demonstrated previously [18]. This combination of parameters is henceforth referred to as "standard force field", to distinguish it from models that attempt to explicitly include specific interactions between adsorbates and OMS. 


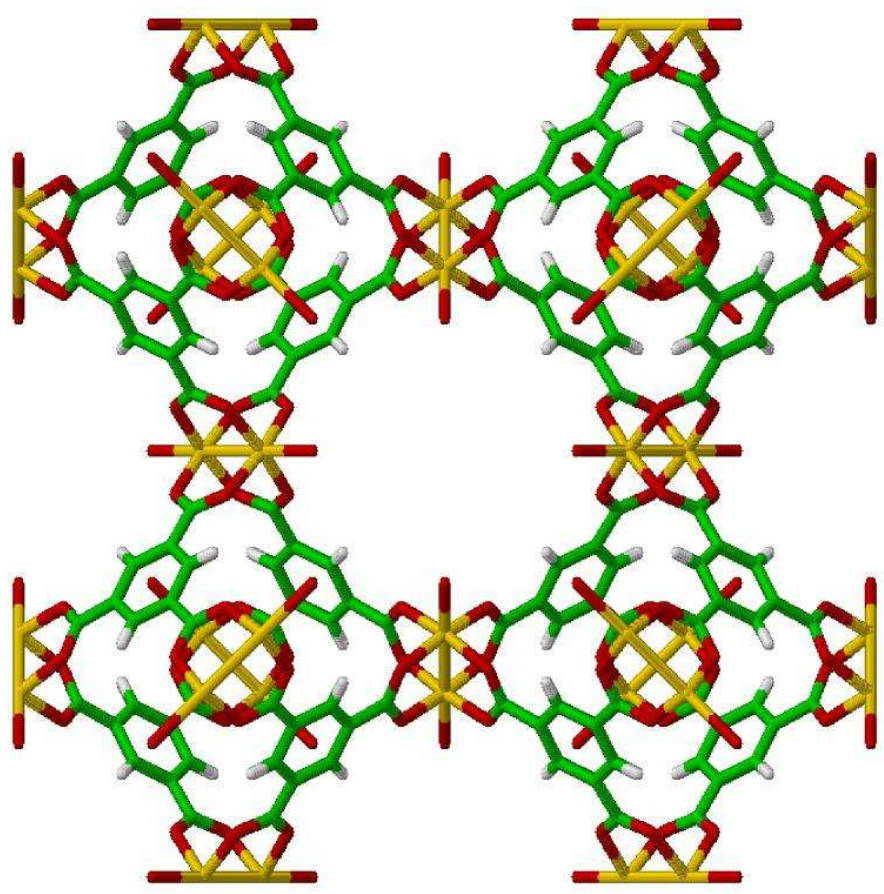

Figure 1 - Diagram of the CuBTC framework. Carbon atoms are green, hydrogen atoms are white, oxygen atoms are red and copper atoms are yellow. Notice the oxygens belonging to water molecules axially coordinated to the $\mathrm{Cu}$ atoms, which are removed during the activation procedure.

Adsorbate molecules were modeled using a united-atom (UA) description, by considering each $\mathrm{CH}_{\mathrm{x}}$ group as a single interaction center with effective potential parameters. With this approach, propane is composed of two $\mathrm{CH}_{3}\left(\mathrm{sp}^{3}\right)$ sites and one central $\mathrm{CH}_{2}\left(\mathrm{sp}^{3}\right)$ site, and propylene contains one $\mathrm{CH}_{3}\left(\mathrm{sp}^{3}\right)$, one $\mathrm{CH}\left(\mathrm{sp}^{2}\right)$ and one $\mathrm{CH}_{2}\left(\mathrm{sp}^{2}\right)$ site. $\mathrm{LJ}$ parameters for $\mathrm{sp}^{3}$ sites were taken from the work of Dubbeldam et al. [36], while those for $\mathrm{sp}^{2}$ sites were taken from Jakobtorweihen et al. [37]. These parameters result from a reparameterization of the standard TraPPE-UA force field $[38,39]$ for use in adsorption studies, and provide very good agreement with experimental adsorption data of olefins and paraffins in zeolites [36,37,40,41]. All bond lengths and angles were considered to be rigid. We have compared adsorption isotherms obtained with rigid and flexible propane molecules, and found the results to be statistically indistinguishable. Interactions between different sites were computed from the standard Lorentz-Berthelot combining rules, and all interactions were cut off at a distance of 1.3 $\mathrm{nm}$. All adsorbate sites were considered to be electronically neutral, which means that electrostatic interactions were neglected. This is a common assumption when modeling hydrocarbons using a UA approach, and has yielded excellent results in the past [36,37,40,41]. Nevertheless, the implications of this assumption for olefin/paraffin adsorption in CuBTC will be discussed later.

\subsection{Density Functional Theory calculations}

All-electron DFT calculations were carried out to obtain accurate potential energy profiles for the interaction between the adsorbates and the OMS of CuBTC. They were performed using the $\mathrm{DMol}^{3}$ code $[42,43]$ included in the Accelrys "Materials Studio" package [44]. A "double numerical plus polarization" (DNP) basis set was employed in all calculations. The PBE exchange-correlation 
functional was used [45], as it was found in earlier studies that this functional predicts reasonable equilibrium distances and interaction energies for the adsorption of hydrogen and acetylene at unsaturated copper sites [24,25]. Calculations were carried out for both ethane and ethylene interaction with $\mathrm{Cu}$ sites in different $\mathrm{Cu}_{2}(\mathrm{~L})_{4}$ clusters, where $\mathrm{L}$ can be either formate (fa), benzene monocarboxylate (bmc), 1,4-benzene dicarboxylate (bdc) or 1,3,5-benzene tricarboxylate (btc). These clusters were chosen to represent progressively larger and more realistic descriptions of the environment felt by the adsorbate in the CuBTC framework. For bdc and btc, the dangling carboxylate groups were rendered neutral by connecting one of the oxygens to a hydrogen atom. Ethane and ethylene were chosen as the simplest representatives of olefins and paraffins - DFT calculations of propane and propylene were not performed, since this would introduce unnecessary complexity arising from additional non-specific interaction sites, molecular asymmetry and additional orientational degrees of freedom, without necessarily improving the description of the local environment around the $\mathrm{Cu}-\pi$ interaction.

As a generic approach, the geometries of the isolated adsorbate molecules and of the isolated $\mathrm{Cu}_{2}(\mathrm{~L})_{4}$ clusters were initially optimized, and the corresponding optimized geometries and energies were taken as reference points for calculating the interaction energy curves. Afterwards, the two optimized fragments were combined, with the adsorbate placed directly above the $\mathrm{Cu}$ atom - i.e., the center of mass $(\mathrm{COM})$ of the adsorbate was located in the direction of the $\mathrm{Cu}-\mathrm{Cu}$ vector, and the adsorbate $\mathrm{C}-\mathrm{C}$ bond was perpendicular to this vector, as shown in Figure 2. The total energy of the complex was then calculated for different values of the distance between the $\mathrm{Cu}$ atom and the $\mathrm{COM}$ of the adsorbate. An example of the calculation setup for ethylene adsorbed on $\mathrm{Cu}_{3}(\mathrm{btc})_{2}$ is shown in Figure 2 (other cluster geometries are given in Supporting Information). For each value of this distance $(r)$, ranging from 0.2 to $0.5 \mathrm{~nm}$, the interaction energy $\left(U_{D F T}\right)$ was computed from equation 1 :

$$
U_{D F T}(r)=U_{\text {Complex }}(r)-U_{\mathrm{Cu}_{2}(L)_{4}}-U_{A d s o r b a t e}
$$

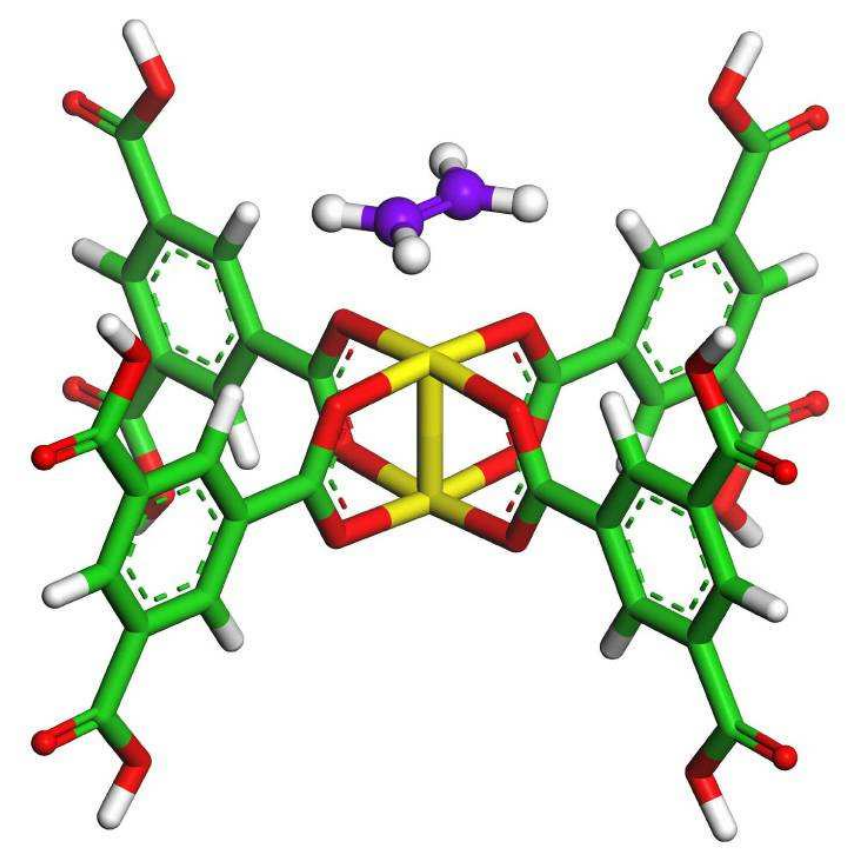


Figure 2 - Geometry used for the DFT calculations of ethylene adsorbing onto a $\mathrm{Cu}_{3}(\mathrm{btc})_{2}$ cluster. Color coding for the framework atoms is the same as in Figure 1, while the ethylene carbon atoms are shown as purple spheres.

In the majority of cases, $U_{\text {Complex }}$ was computed from single-point calculations at different values of $r$, considering different orientations of the adsorbate, but in some cases selected structural degrees of freedom were allowed to relax, keeping $r$ constant. These partially optimized systems included full optimization of the adsorbate molecule while keeping the cluster rigid, as well as optimization of both the adsorbate and the $\mathrm{Cu}-\mathrm{Cu}$ distance in the cluster while keeping the remaining cluster atoms fixed. In the latter case, we found that when only one ethylene molecule adsorbed in the vicinity of a $\mathrm{Cu}$ atom, the cluster was significantly distorted, with both $\mathrm{Cu}$ atoms moving in the direction of the adsorbate. To avoid this excessive distortion of the cluster (which is unrealistic, given that such deformations are suppressed in the periodic structure of the real CuBTC crystal [46]), in this particular case two $\mathrm{C}_{2} \mathrm{H}_{4}$ molecules were adsorbed simultaneously and symmetrically, one on each side of the cluster. The effect of adding a second adsorbate molecule on the DFT interaction energy per molecule was tested, and was found to be negligible. The effects of the size of the cluster, the orientation of the adsorbate and the degree of optimization will be discussed in detail in section 3 .

\subsection{Specific interactions with Open Metal Sites}

Once the interaction energy profiles as a function of $r$ are calculated with DFT, as described in section 2.2, they can be used to include a description of the specific olefin-Cu interactions into the standard force field for use in GCMC simulations. An important problem immediately arises from the fact that most used DFT exchange-correlation functionals (local and semilocal approaches) are not able to take into account dispersion dominated interactions, with strong efforts still being made to solve this issue [47-52]. However, dispersion interactions are usually dominant in adsorption systems, and the present system is no exception. Another apparent difficulty comes from our assumption of neutral adsorbates in GCMC, thus neglecting Coulombic interactions altogether, which is in contrast with the electrostatically-dominated DFT calculations. To simplify the problem, we begin by assuming that the DFT energies are the result of only two contributions to the adsorption energy repulsion and specific interactions between $\mathrm{Cu}$ atoms and the $\pi$ orbitals of unsaturated $\mathrm{C}=\mathrm{C}$ bonds. In other words, we assume that the dispersion contribution is precisely zero in the DFT calculations, while any non-specific electrostatic interactions between ethylene and the cluster, if existent, are implicitly included into the above two contributions. Although these assumptions may appear to be quite strong, we will show below that excellent predictions of adsorption are obtained. The validity of these assumptions, and possible approaches for lifting them, will be discussed in Section 3.4.

With the above assumptions, the specific $\mathrm{Cu}-\pi$ interaction can be computed from:

$$
U_{C u-\pi}(r)=U_{D F T}(r)-U_{R e p}(r)
$$

This means that we need to find a way of estimating the repulsive contribution to the adsorption energy in order to isolate the specific contribution of the OMS. Ideally, $U_{R e p}$ should be as close as possible to the repulsion actually experienced by the adsorbate in the model framework during the GCMC simulations. We achieve this by applying the Weeks-Chandler-Andersen (WCA) approach [53] to separate the repulsive and the attractive contributions of the Lennard-Jones potential. The repulsive WCA potential is given by: 


$$
U_{\text {Rep }}(r)= \begin{cases}U_{L J}(r)-U_{L J}\left(r_{\min }\right) & r<r_{\min } \\ 0 & r \geq r_{\min }\end{cases}
$$

Essentially, it truncates the LJ potential at its minimum point and then shifts the curve upward by the energy value at that minimum. The result is a purely repulsive curve that dies out to zero precisely at the point where the LJ potential reaches a minimum (see red line in Figure 3). In practice, the adsorbate was placed at successively increasing values of $r$ and the repulsive interaction was computed using the standard force field and the WCA approximation, taking the adsorbate orientation into account by applying a Monte Carlo procedure - a very large number of different random orientations of the adsorbate were sampled for each $r$, and the minimum value of the energy sampled was chosen as the value of $U_{R e p}$ at that value of $r$. This procedure ensures that during the GCMC with the modified potential the adsorbate does not get trapped in any unphysical configuration due to an inadequate balance between the repulsion that is present in the standard force field and the newly introduced attractive $\mathrm{Cu}-\pi$ interaction. In preliminary tests where the repulsive contribution was estimated without sampling over adsorbate orientations (i.e., keeping the ethylene orientation fixed and equal to the DFT calculations), several olefin molecules adopted unphysical configurations, e.g., parallel to the $\mathrm{Cu}-\mathrm{Cu}$ vector, with the center of the $\mathrm{C}=\mathrm{C}$ double bond overlapping with one of the $\mathrm{Cu}$ atoms.

By subtracting the repulsive curve thus calculated from the original DFT curve, we can obtain an estimate of the actual (fully attractive) $\mathrm{Cu}-\pi$ interaction as a function of $r$. This curve, shown as black circles in Figure 3, was then fitted to an appropriate functional form and included into the GCMC calculations. Strictly speaking, the $\mathrm{Cu}-\pi$ profile for a single adsorbate molecule contains contributions from its interaction with both $\mathrm{Cu}$ atoms of the cluster, according to equation 4 :

$$
U_{C u-\pi}(r)=U_{\text {Function }}(r)+U_{\text {Function }}\left(r+d_{C u-C u}\right)
$$

where $d_{\mathrm{Cu}-\mathrm{Cu}}$ is the distance between the two copper atoms in the cluster. In practice, the contribution of the second $\mathrm{Cu}$ atom to the interaction energy is very small, but it was included in the fitting procedure for consistency.

Finally, we need to define the form of the fitting function. We have chosen to use a Morse potential to describe the underlying attractive well, plus a power law to capture the monotonically decreasing character of the resulting curve (see Figure 3). Equation 5 describes the full fitting function, where it should be noted that the interaction represented by this function occurs between a $\mathrm{Cu}$ atom and a new site located at the center of the $\mathrm{C}=\mathrm{C}$ bond. In this equation, $R_{0}$ is a distance parameter correponding to the position of the minimum of the Morse potential, $D_{0}$ is the energy value at that minimum (these two parameters are analogous to those of the LJ potential), $\alpha$ is a stiffness parameter that adds flexibility to the Morse function, while $A$ and $B$ are empirical parameters of the power law expression.

$$
U_{\text {Function }}\left(r_{i j}\right)=D_{0}\left\{\exp \left[\alpha\left(1-\frac{r_{i j}}{R_{0}}\right)\right]-2 \exp \left[\frac{\alpha}{2}\left(1-\frac{r_{i j}}{R_{0}}\right)\right]\right\}+\left(\frac{A}{r_{i j}}\right)^{B}
$$

An example of the fitting procedure is shown in Figure 3. The repulsion contribution (red line) is subtracted from the original DFT curve (solid circles in the inset) to obtain the underlying $\mathrm{Cu}$ $\pi$ interaction (solid circles in the main panel), which is then fitted to equation 5 (full black line). The 
fit is divided into its two separate contributions to show that the approach yields physically reasonable parameters. The fit to the DFT-derived data is very good, which can be seen directly from the $\mathrm{Cu}-\pi$ interactions (compare the circles to the full black line in the main panel of Figure 3) or recovering the DFT curve by adding the fit to the WCA repulsive contribution (comparison in the inset of Figure 3 ). We note here that although a very good fit was obtained using equation 5 , this function is by no means a unique way of describing this type of interaction. For example, an orientationally-dependent potential may yield a more realistic description of $\mathrm{Cu}-\pi$ interactions. However, such an approach was not pursued here since it would significantly increase the complexity of the calculations.

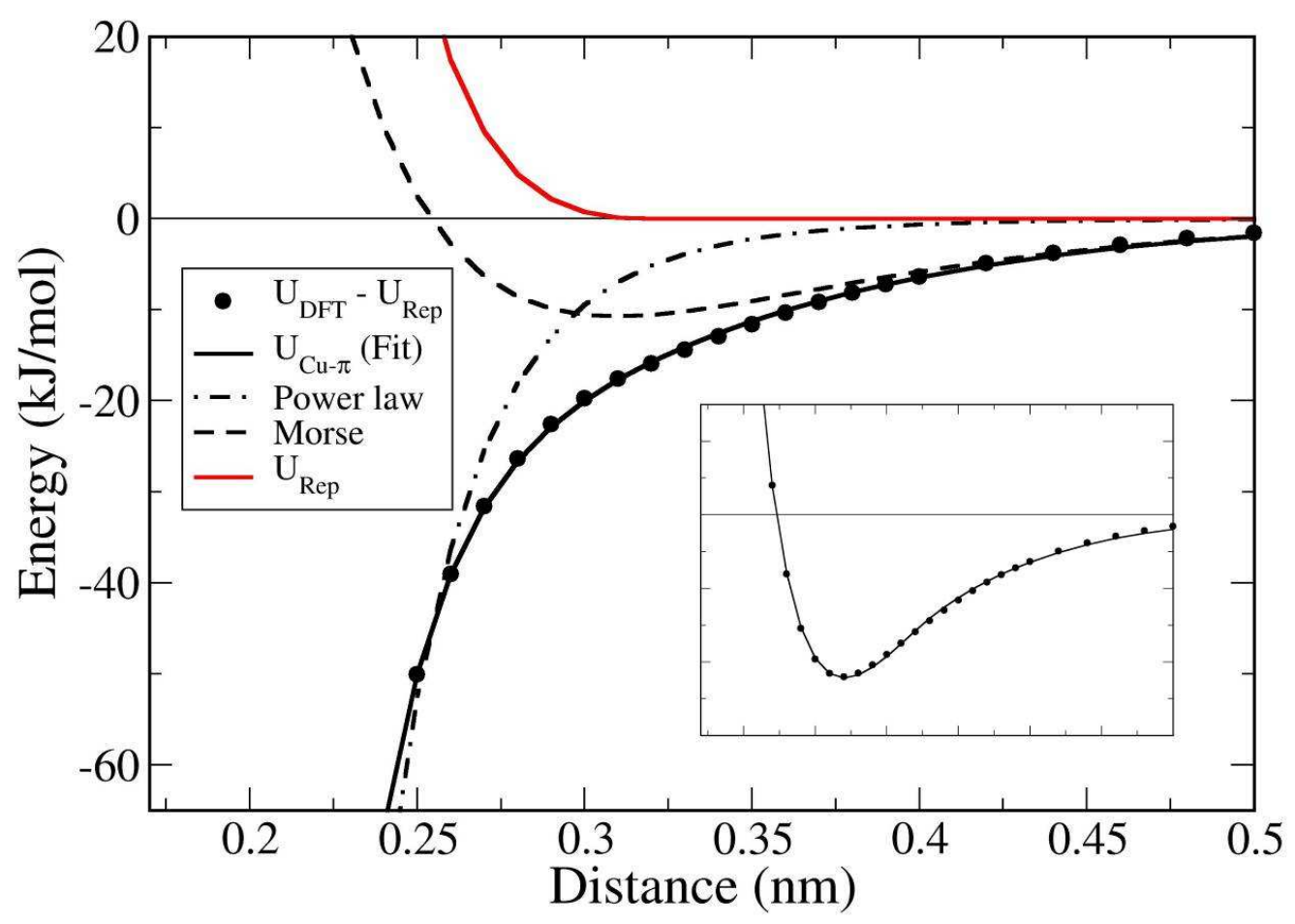

Figure 3 - Fit to the $\mathrm{Cu}-\pi$ interaction energy. Circles are data obtained from the DFT profile (inset) subtracting the repulsive component (red line) given by equation 3, while the full black line is the fit to those points using equations 4 and 5 . The dashed and dotted-dashed lines represent the two separate contributions to the fitting function, from the Morse potential and the power law, respectively. The inset shows the original DFT profile together with the curve obtained by adding the $\mathrm{Cu}-\pi$ fit to the WCA repulsive potential.

The curve obtained from the fit is added to the calculation of the olefin-MOF interaction in each step of the GCMC procedure. Because the reference point for the interaction is the mid-point of the $\mathrm{C}=\mathrm{C}$ bond, an extra interaction site was added to propylene, located precisely between the two $\mathrm{sp}^{2}$ sites. The computational overhead of adding an extra site is quite small. As the nature of the $\mathrm{Cu}-\pi$ interaction is intrinsically short-ranged, a cutoff of $0.5 \mathrm{~nm}$ was used for the calculation of this interaction in GCMC. DFT calculations beyond this distance become less reliable. The adsorbateadsorbate interactions were not changed in the modified potential. 


\section{3 - Results and discussion}

\subsection{Performance of standard force fields}

To begin with, we check the performance of the standard force-field described in section 2.1 for predicting propane and propylene adsorption in CuBTC. In Figure 4 we compare pure component simulated and experimental adsorption for these systems at a temperature of $323 \mathrm{~K}$ (results at other temperatures were analogous). The simulations for propane slightly overestimate the experimental data in the entire range of pressures. This effect could be due to the fact that GCMC simulations assume a perfect crystalline framework, whereas the real material may contain defects, residual solvent and/or pore blockages that would render part of the pore volume unavailable for adsorption. We have accounted for this effect by rescaling the simulated adsorbed amount by a constant value (without modifying the force field parameters) [19]. We find that a factor of 0.84 gives the best agreement between simulation and experiment (see dashed black line in Figure 4). Although a "dead volume" of $16 \%$ is physically reasonable, the overestimation could also be due to other effects, including an inadequacy of the force-field, which we cannot presently rule out. This will be discussed in more detail later, but for the time being, we shall assume that this percentage of excluded volume applies to all subsequent simulated isotherms (for all adsorbates, all temperatures, all pressures and all compositions). Figure 5 shows that excellent agreement between experiments and simulations using this uniform scaling factor is obtained for propane at several temperatures.

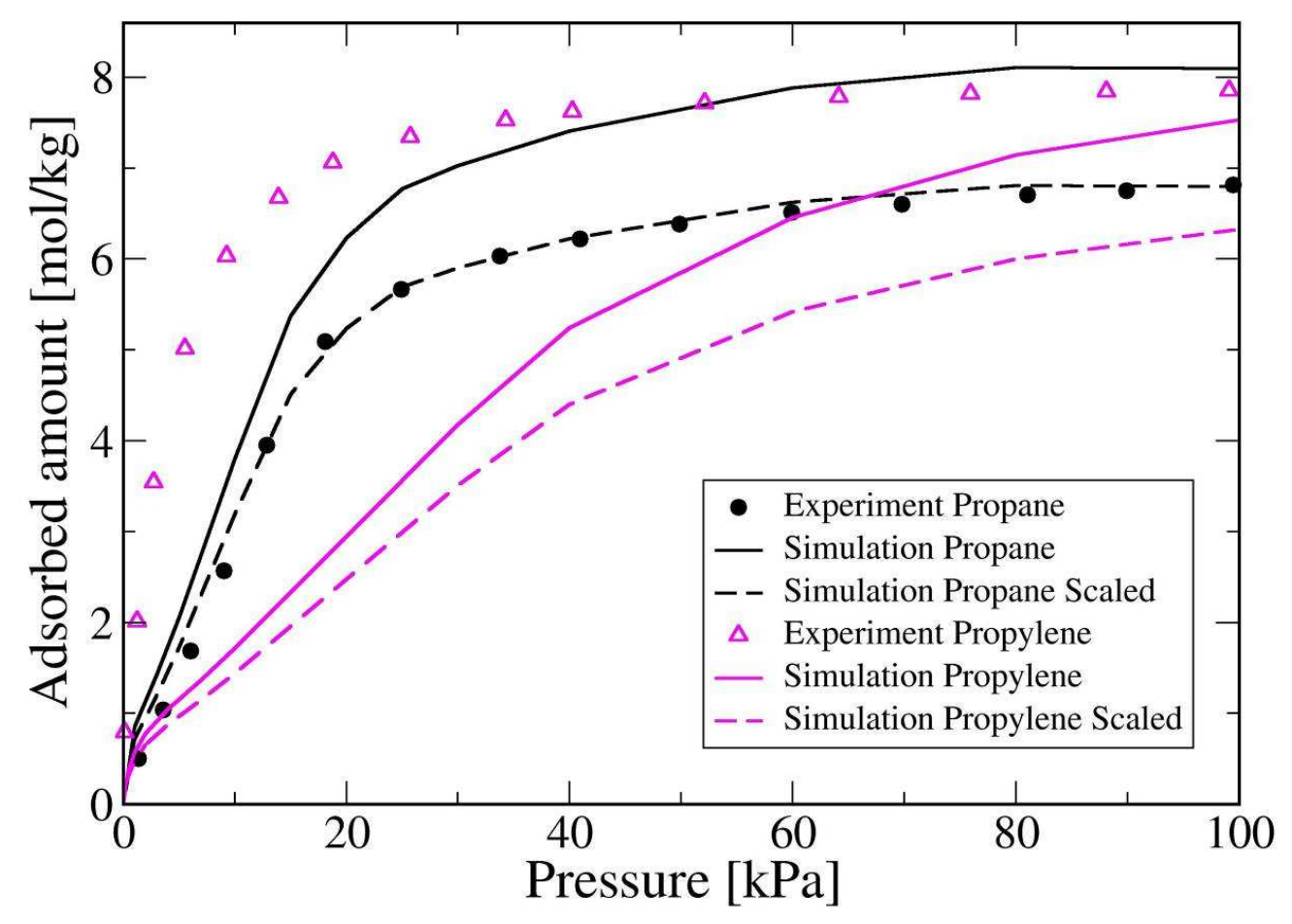

Figure 4 - Comparison between experimental adsorption isotherms (points) and simulations using the DREIDING force-field (full lines), for propane (solid circles and black lines) and propylene (open triangles and magenta lines) on $\mathrm{CuBTC}$ at $323 \mathrm{~K}$. The dashed lines represent the simulation results 
scaled by a factor of 0.84 , to account for possible pore blocking, defects and impurities in the sample material. Experimental data are from Lamia et al. [22].

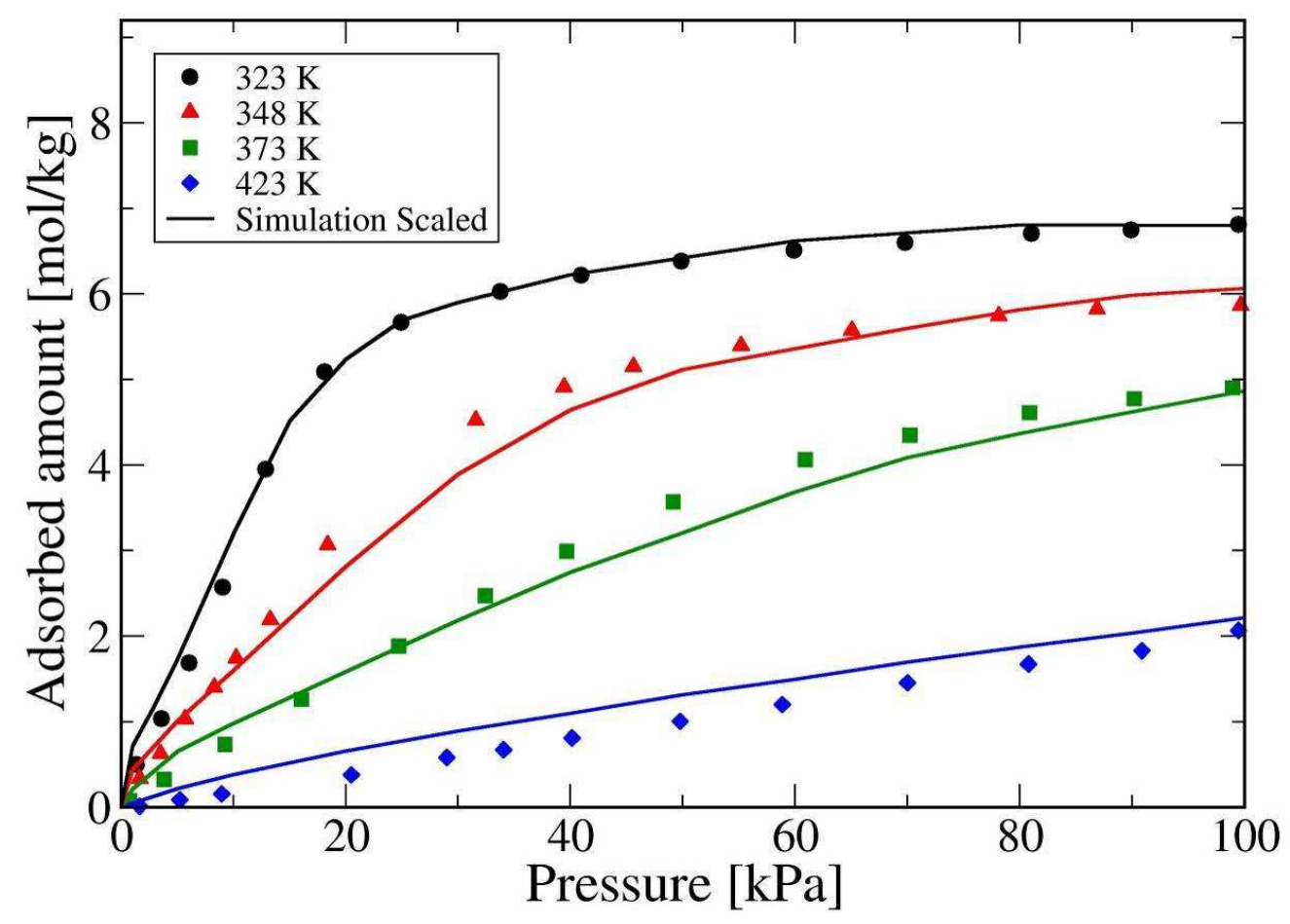

Figure 5 - Comparison between experimental adsorption isotherms (points) and simulation results scaled by a factor of 0.84 (full lines), for propane on CuBTC at several temperatures. Experimental data are from Lamia et al. [22].

If we now compare the isotherms for propylene on the same material (magenta lines in Figure 4), we observe that the simulations dramatically underestimate the amount adsorbed (naturally, scaling the simulated isotherm only makes matters worse). This has been observed previously [22], and is due to an inability of standard force fields to describe specific interactions between the unsaturated $\mathrm{Cu}$ atom and the $\pi$-orbitals of alkenes. Our main concern during the remainder of this paper will be to present a consistent framework for the incorporation of these interactions into a classical molecular model for adsorption predictions.

The shortcomings of standard force-fields for dealing with this type of system can be further understood by analyzing DFT adsorption profiles for both olefins and paraffins. In Figure 6 we plot DFT curves (black lines) for ethane and ethylene adsorbing over the $\mathrm{Cu}$ atom of $\mathrm{a} \mathrm{Cu}_{2}(\mathrm{btc})_{4}$ cluster with all geometries fixed to those of the corresponding optimized isolated fragments, showing the interaction energy as a function of distance between the center of the double bond and the $\mathrm{Cu}$ atom. These are compared to analogous profiles obtained using the DREIDING model (magenta curves), which accounts only for repulsive and dispersive interactions via a Lennard-Jones potential. The "classical" profiles are very similar for both molecules, reflecting the similarity in their geometry and LJ parameters. Conversely, the DFT profiles for ethylene show a much stronger interaction with the cluster than for ethane - the former shows a deep attractive well at a distance of about $0.27 \mathrm{~nm}$ while the latter shows a very shallow well at around $0.35 \mathrm{~nm}$. The potential well for ethylene is clearly not 
due solely to dispersion interactions, as it is much deeper and occurs at much shorter distances to the metal than the LJ potential well. Instead, it reflects the $\pi$-donation of electrons from the olefin to the vacant p-orbital of the metal, together with back-donation from d-orbitals of the metal to the antibonding $\pi^{*}$-orbital of the olefin [54]. As expected, these strong specific interactions between the ethylene double bond orbitals and the $\mathrm{Cu}$ atom are not captured by the DREIDING potential.

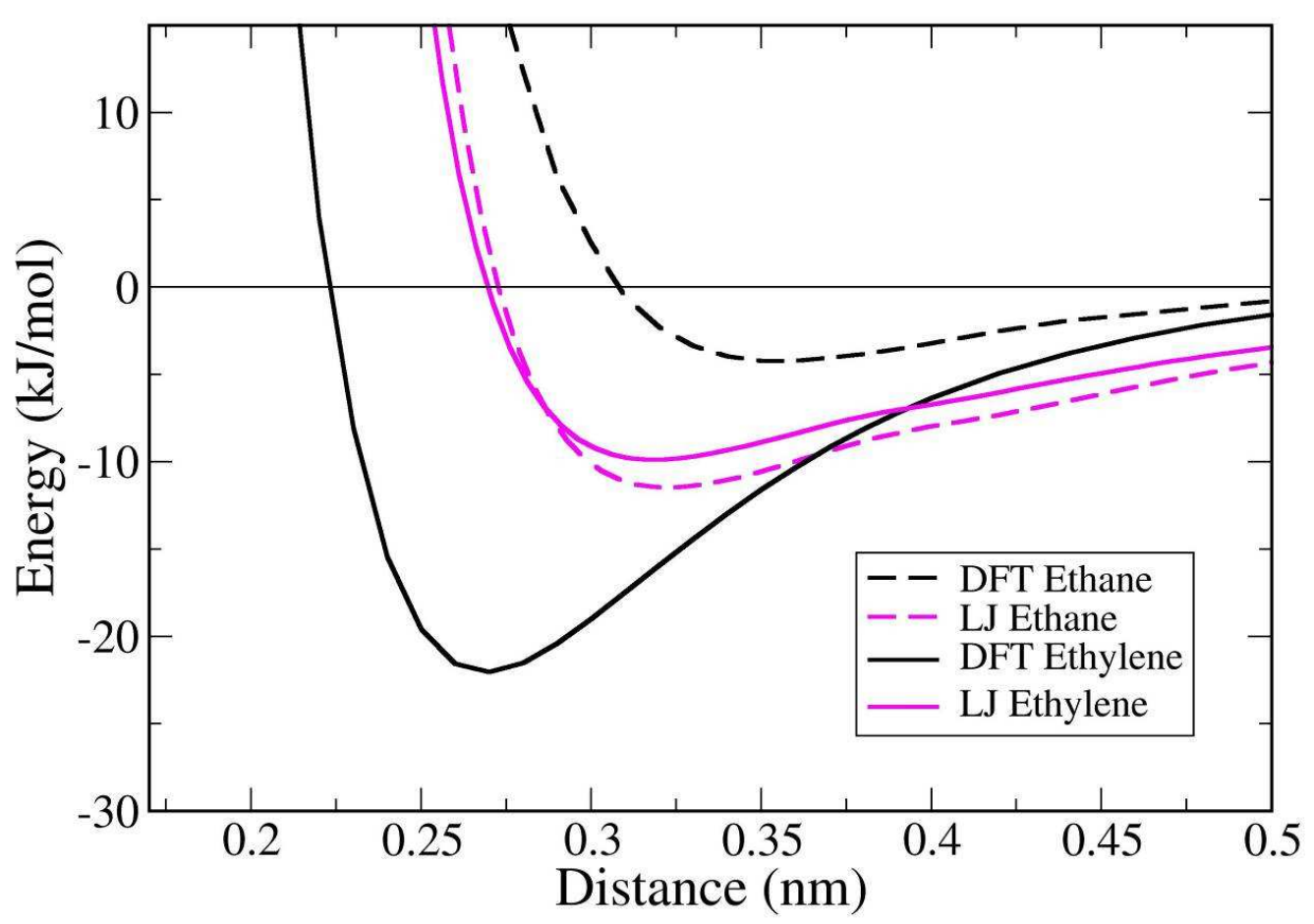

Figure 6 - Potential energy scans for ethane (dashed lines) and ethylene (full lines) adsorbed on a $\mathrm{Cu}_{2}(\mathrm{btc})_{4}$ cluster. Black curves were obtained with DFT, while magenta curves were obtained using the classical DREIDING force-field. DFT scans were obtained from single-point calculations on previously optimized fragments.

It is also interesting to compare the DFT and classical curves for ethane (dashed lines in Figure 6). In this case the DFT well is much shallower than the LJ well, which is due to the wellknown difficulty of DFT methods for accurately quantifying dispersion interactions [47-52]. Nevertheless, some degree of interaction is still captured by DFT, for which we can think of several possible explanations: i) this particular combination of density functional and basis set is able to capture a small degree of dispersion interactions; ii) the curve reflects a very mild specific interaction between ethane and the open metal site; iii) the well is due to mild non-specific electrostatic interactions, which are only implicitly accounted for in our model. At this moment, we are not able to determine which of those alternatives (or combination thereof) is the correct one. We are currently undertaking more detailed ab initio calculations for these systems, using a much higher level of theory (MP2 energies extrapolated to the infinite basis set limit), to clarify this issue. For the time being, we will maintain our initial assumption that the DFT energy can be divided into a repulsive and a specific $\mathrm{Cu}-\pi$ contribution (equation 2). 


\subsection{Choice of DFT protocol}

As mentioned in section 2, in order to address the limitations of standard force fields for describing specific interactions between olefins and OMS, we make use of DFT interaction energy profiles (such as those shown in Figure 6) to calibrate a new molecular model. The first step in this approach, therefore, is the choice of DFT protocol to calculate the potential energy profiles. We begin by testing the effect of the cluster size on the calculated profiles, comparing results obtained on rigid $\mathrm{Cu}_{2}(\mathrm{~L})_{4}$ clusters, with $\mathrm{L}=\mathrm{fa}$, bmc, bdc and btc. These curves are shown in Figure 7 .

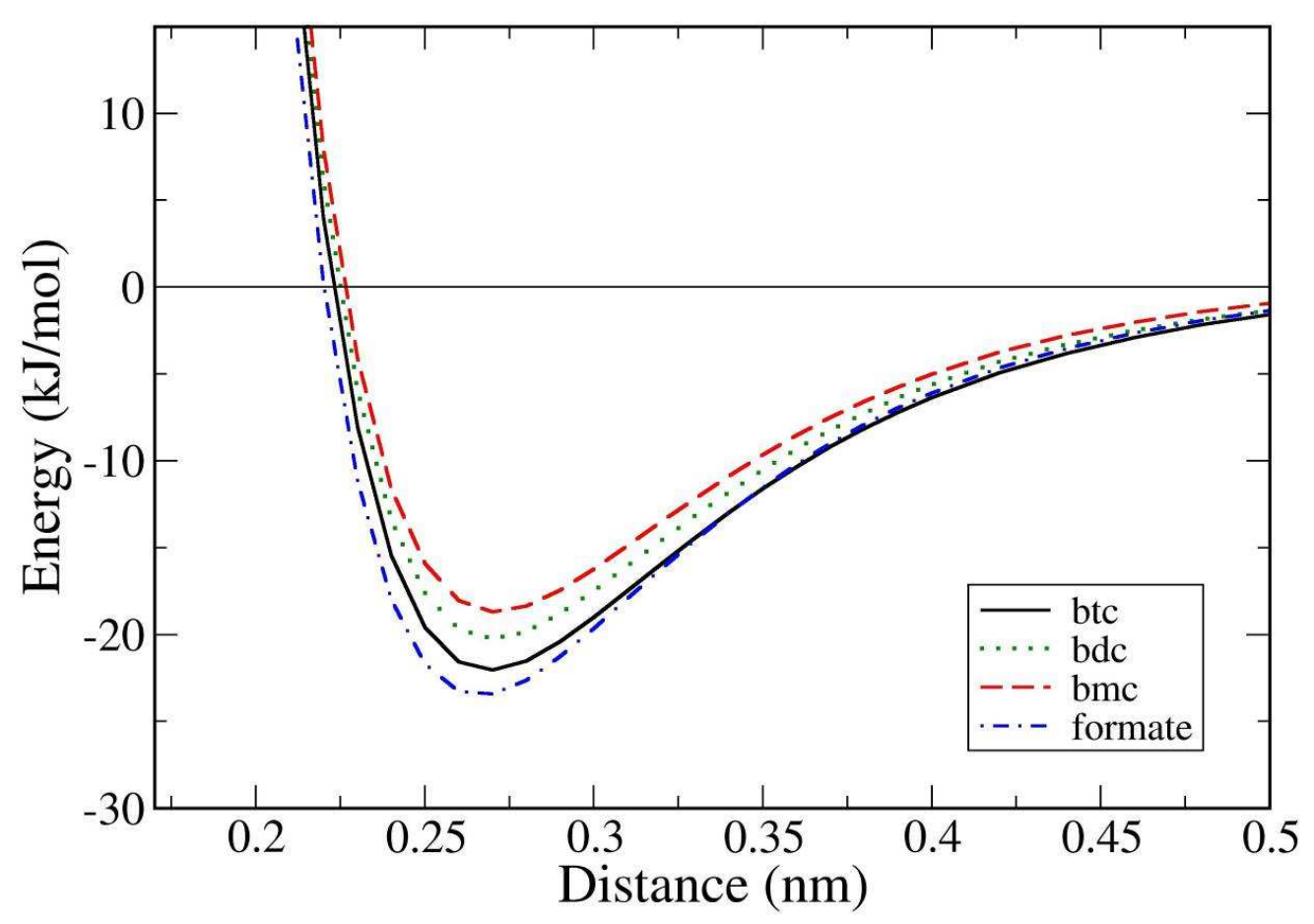

Figure 7 - Potential energy scans for ethylene adsorbed on a rigid cluster using DFT with the PBE functional and different cluster sizes: 1) formate - blue dashed-dotted line; 2) benzenemonocarboxylate (bmc) - red dashed line; 3) benzene-dicarboxylate (bdc) - green dotted line; 4) benzene-tricarboxylate (btc) - full black line.

The size of the framework cluster has a measurable effect on the depth of the $\mathrm{Cu}-\pi$ potential well, but a negligible effect on its location. The progressive addition of carboxyl groups on the benzene rings connected to the $\mathrm{Cu}$ dimer (see Figures S2, S3 and 2) cause a progressive deepening of the well (compare curves for bmc, bdc and btc in Figure 7). This is due to an increase in the electronwithdrawing power (inductive effects) of the substituents in this series, which contributes to further stabilize the adsorbate-cluster complex, as observed previously [55]. Interestingly, the curve for formate is rather close to the more realistic btc cluster (which is most similar to the actual structure of the MOF framework). This suggests that the $\mathrm{Cu}_{2}(\mathrm{fa})_{4}$ cluster may actually be a reasonable model for this type of interaction, at a much lower computational cost. For the particular combination of functional and basis set used in this work, computational cost is not a major issue, so we have carried out all further calculations with the more realistic $\mathrm{Cu}_{2}(\mathrm{btc})_{4}$ cluster. However, if much higher-level methods are applied, in which case computational cost will become an important variable, the $\mathrm{Cu}_{2}(\mathrm{fa})_{4}$ 
cluster may present a useful alternative. Finally, it is important to note that previous studies suggest that adsorption energies on OMS obtained from small molecular clusters are a good approximation to more accurate calculations in a periodic structure [56].

If we fit equation 5 to the DFT curves from Figure 7 and incorporate the $\mathrm{Cu}-\pi$ interactions into the GCMC simulations, following the procedure described in section 2.3, we obtain the adsorption isotherms for propylene shown in Figure 8. The inclusion of these specific interactions into the molecular model yields isotherms that are in much better agreement than those obtained using the standard force field (Figure 5), but this issue will be analyzed in more detail below. For the moment, we concentrate on the effect of the cluster size on the adsorption isotherms. As expected from the profiles shown in Figure 7, the isotherms for formate and btc are almost overlapping, which reinforces our claim that the former is a useful model for these systems. The curves for bdc (not shown) and bmc underestimate the amount adsorbed at both temperatures in the entire pressure range. Based on these results, we do not recommend the use of bmc or bdc clusters for modeling $\mathrm{Cu}-\pi$ interactions.

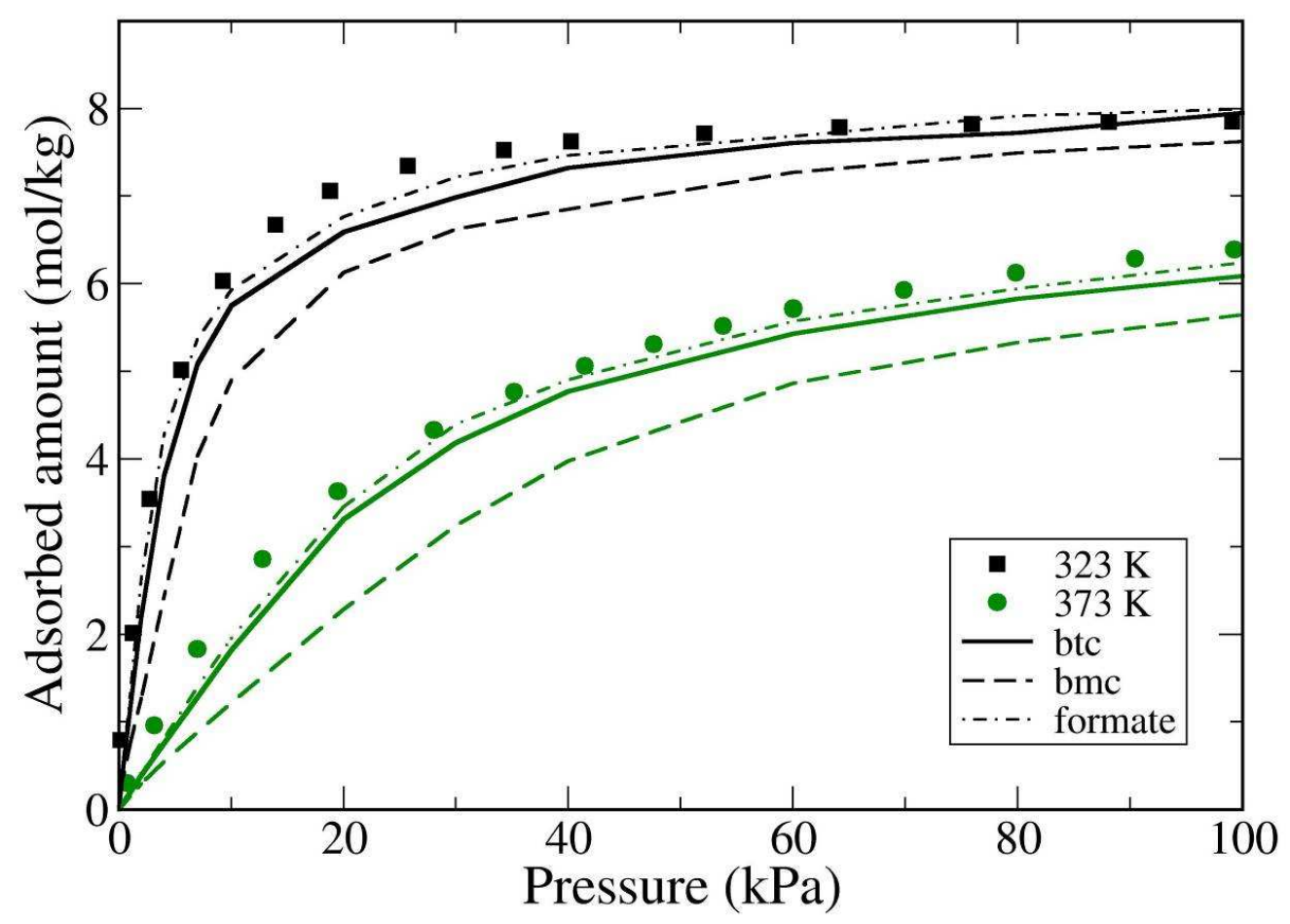

Figure 8 - Comparison between experimental adsorption isotherms (points) and simulation results (lines), for propylene on CuBTC at $323 \mathrm{~K}$ (black) and $373 \mathrm{~K}$ (green). All simulations included the Cu$\pi$ interaction, and were scaled by a factor of 0.84 . The lines correspond to results obtained using DFT data from clusters of different size: 1) formate - dashed-dotted line; 2) benzene-monocarboxylate (bmc) -dashed line; 3) benzene-tricarboxylate (btc) - full line. The curve for bdc is intermediate between btc and bmc, and is not shown for clarity. Experimental data are from Lamia et al. [22].

The second variable we analyzed was the optimization protocol during the DFT calculations. In Figure 9 we compare interaction energy scans obtained with different protocols. Comparing the red and blue curves, obtained with fully fixed geometries (i.e., single-point calculations on previously optimized individual fragments) we see that the ethylene molecule always prefers to be positioned 
directly above the $\mathrm{O}-\mathrm{Cu}-\mathrm{O}$ axis of the framework cluster, to maximize the interactions with those two oxygen atoms. Therefore, as expected, the profile obtained when the intramolecular geometry parameters of the ethylene molecule are fully relaxed during every step of the calculation (green line in Figure 9) is practically identical to the red line.

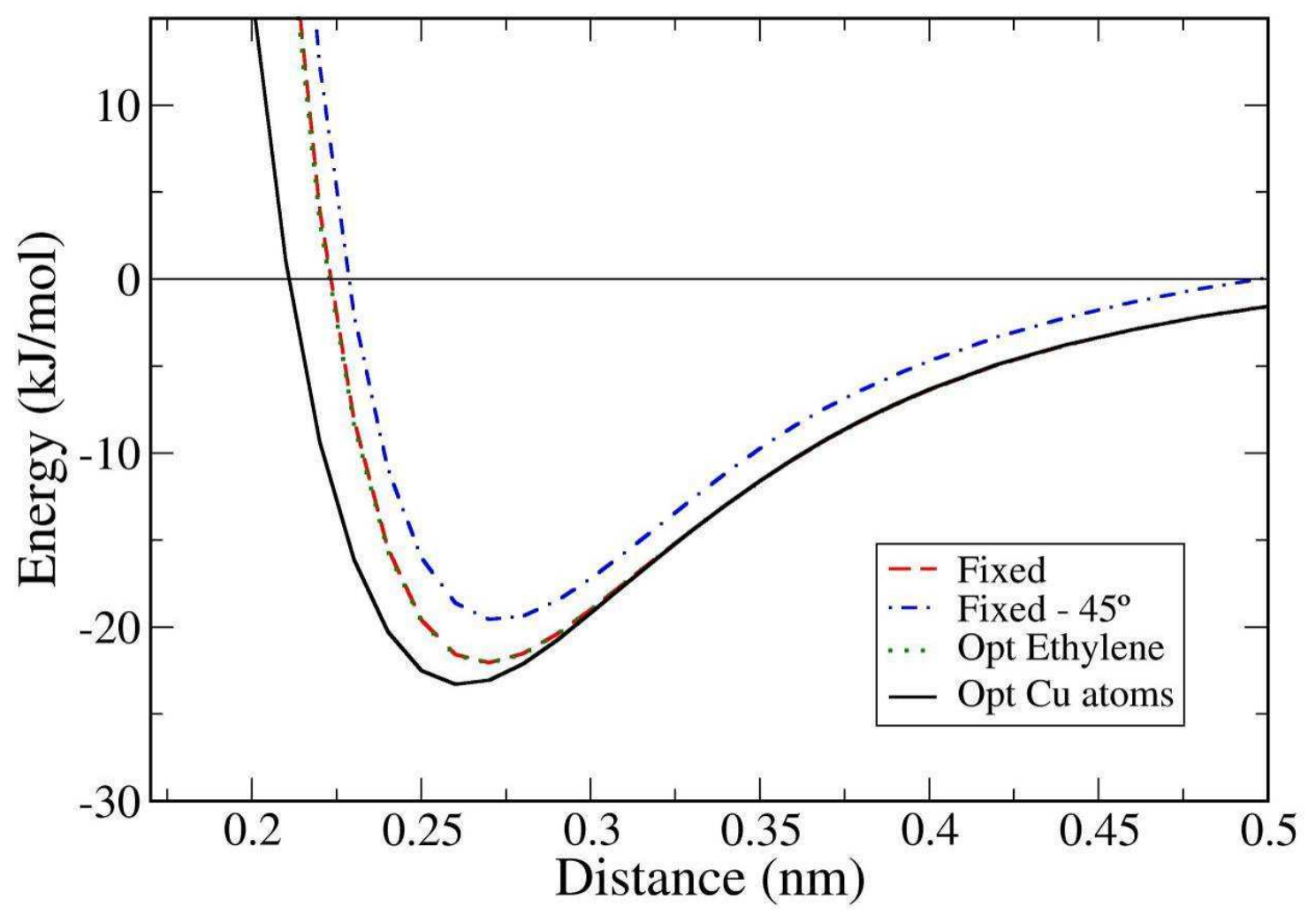

Figure 9 - Potential energy scans for ethylene adsorbed on a $\mathrm{Cu}_{2}(\mathrm{btc})_{4}$ cluster using DFT with the PBE functional and different optimization protocols: 1) fixed, pre-optimized geometries of ethylene and $\mathrm{Cu}_{2}(\mathrm{btc})_{4}$, with ethylene positioned directly above the O-Cu-O axis - red dashed line; 2) fixed, pre-optimized geometries of ethylene and $\mathrm{Cu}_{2}(\mathrm{btc})_{4}$, with ethylene positioned at a $45^{\circ}$ angle to the O$\mathrm{Cu}-\mathrm{O}$ axis - blue dotted-dashed line; 3) fixed, pre-optimized geometry of $\mathrm{Cu}_{2}(\mathrm{btc})_{4}$, with the ethylene molecule allowed to relax - green dotted line; 4) fixed, pre-optimized geometry of $\mathrm{Cu}_{2}(\mathrm{btc})_{4}$, with the ethylene molecule and the $\mathrm{Cu}$ atoms allowed to relax - full black line.

A significant difference is observed when the the intramolecular geometry parameters of the ethylene molecule and the $\mathrm{z}$ coordinates of the $\mathrm{Cu}$ atoms are also optimized. Indeed, the potential well becomes slightly deeper and shifts to slightly shorter distances compared to the curve obtained from fixed fragments. More importantly, the repulsive region of the curve is shifted to shorter distances, which means that the ethylene molecule is allowed to penetrate closer to the $\mathrm{Cu}$ atoms. Although the change in the DFT profile is significant, the effect of the degree of optimization of the cluster on the actual GCMC isotherms is relatively small (see Figure S4). This is most likely because the region of the curve that is affected (the repulsive part) is not often accessible during the GCMC simulations. Instead, in the majority of sampled configurations, adsorbed molecules will be located in the potential well region, which is reasonably well described by the fixed-cluster profile. Naturally, single-point calculations on fixed geometries are much faster than when the clusters are allowed to optimize. 
Nevertheless, because computational cost is not a major issue in this study, we will use the more realistic $\mathrm{Cu}$-optimized protocol in the remainder of our GCMC calculations.

\subsection{New model for MOFs with OMS}

Using the DFT protocol determined in the previous section $\left(\mathrm{Cu}_{2}(\mathrm{btc})_{4}\right.$ cluster with ethylene and $\mathrm{Cu}$ atoms allowed to relax during the scan), we have applied the procedure outlined in section 2.3 to calculate GCMC adsorption isotherms for propylene on the full CuBTC framework at several temperatures. The parameters obtained from the fit to the DFT profile using equations 4 and 5 are given in Table 1. GCMC isotherms for propylene including the $\mathrm{Cu}-\pi$ interactions are compared to experimental data in Figure 10, where excellent agreement is obtained at all temperatures.

Table 1 - Parameters obtained by fitting equations 4 and 5 to the DFT-derived profile for $\mathrm{Cu}-\pi$ interactions. Length is expressed in $\mathrm{nm}$ and energy in $\mathrm{kJ} / \mathrm{mol}$.

\begin{tabular}{c|c|c|c|c}
$\mathrm{R}_{0}$ & $\mathrm{D}_{0}$ & $\alpha$ & $\mathrm{A}$ & $\mathrm{B}$ \\
\hline 0.3072 & 11.27 & 7.82 & 0.3768 & 9.56
\end{tabular}

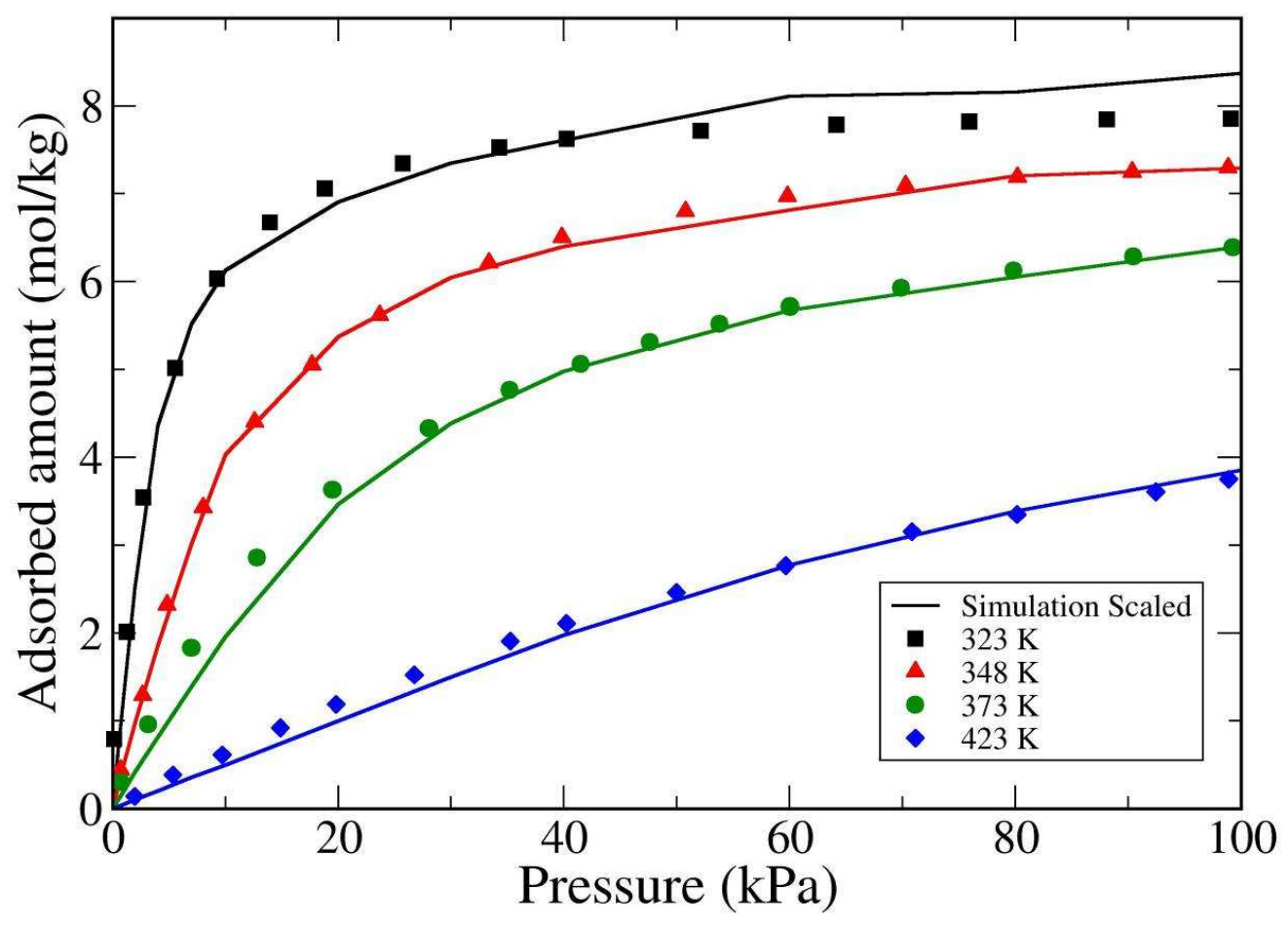

Figure 10 - Comparison between experimental adsorption isotherms (points) and simulation results (full lines), for propylene on $\mathrm{CuBTC}$ at several temperatures. Simulations included the $\mathrm{Cu}-\pi$ interaction, and were scaled by a factor of 0.84 as in the case of propane (Figure 5). Experimental data are from Lamia et al. [22].

Representative snapshots of propylene adsorption, shown in Figure 11, demonstrate that by applying the modified potential, olefin molecules are indeed adsorbing in close vicinity to the OMS of CuBTC. They preferentially align themselves with the $\mathrm{C}=\mathrm{C}$ double bond perpendicular to the $\mathrm{Cu}-\mathrm{Cu}$ 
axis, which corresponds to the most favourable orientation found in the DFT calculations. This gives us confidence that the new model is indeed capturing the correct adsorption mechanism for these systems.
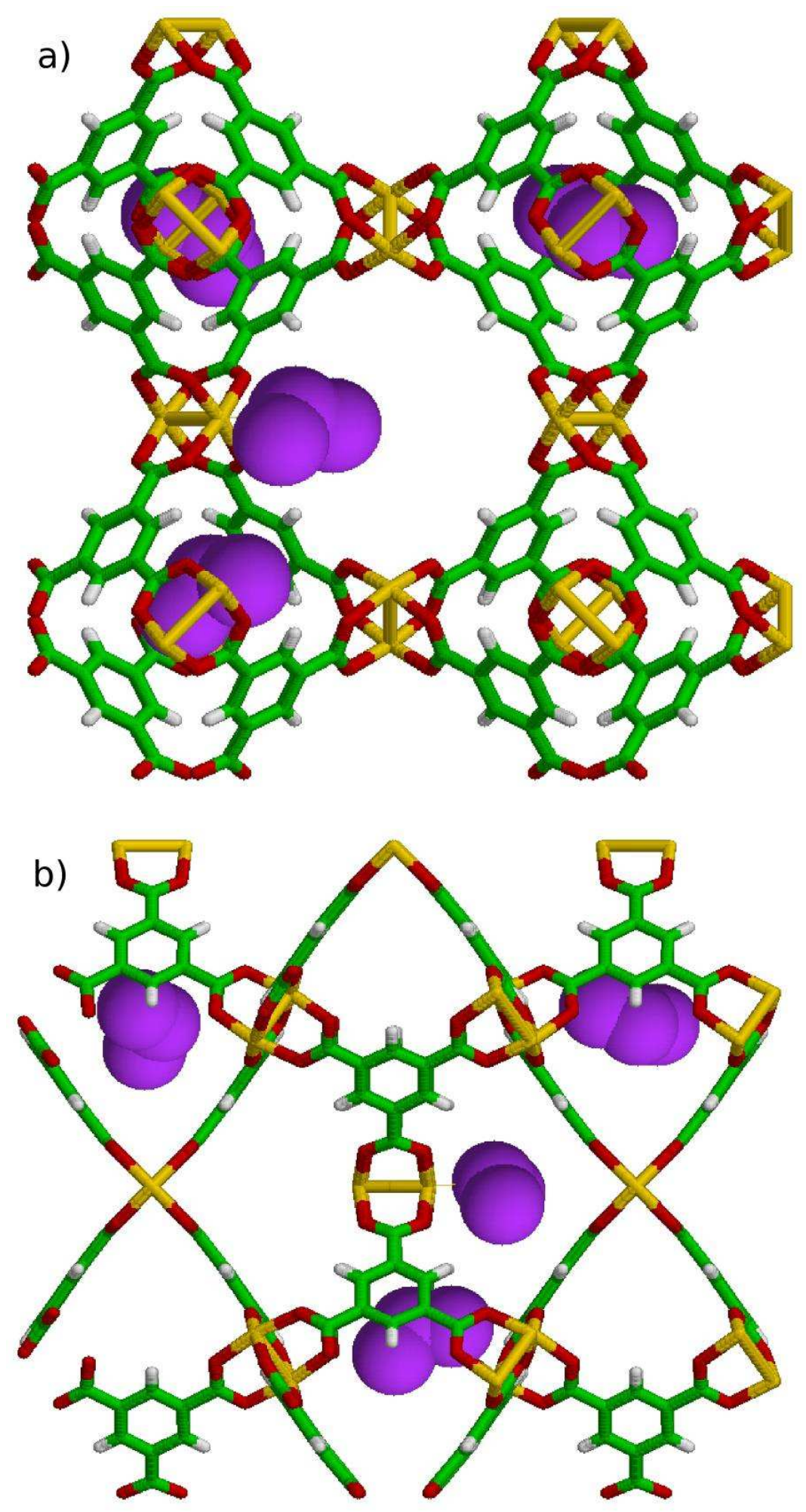
Figure 11 - Typical snapshots of propylene adsorption in $\mathrm{CuBTC}$ at $323 \mathrm{~K}$ and $5 \mathrm{kPa}$, viewed from two different perspectives. Color coding is the same as in Figure 2. Notice the central propylene molecule adsorbing onto the unsaturated $\mathrm{Cu}$ atom.

A more stringent test of the capability of our new model is the prediction of mixture adsorption. In Figure 12, we show adsorption isotherms for binary propane/propylene mixtures of varying bulk-phase composition obtained from GCMC simulations using the new potential model. These are compared to binary isotherms calculated using Ideal Adsorbed Solution Theory (IAST) [57], based on fits of experimental pure-component isotherms to the Dual Site Sips model. The fits to the experimental isotherms and details of the IAST calculation procedure have been given elsewhere $[22,23]$. We can see that there is reasonable qualitative agreement between simulations and IAST (for instance, the crossover point between the propane and propylene isotherms is well predicted), but there are some quantitative discrepancies in the propylene-rich part of the isotherms. Moreover, while IAST predicts a constant selectivity in the entire composition range, our simulations predict increasing selectivity with propane mole fraction (see Figure S5). These differences can be due to three possible reasons: i) inadequacy of the IAST model for describing adsorption in these systems; ii) inadequacy of the DREIDING force-field, with corresponding errors in both propane and propylene adsorption; iii) inadequacy of our approach to account for the $\mathrm{Cu}-\pi$ interactions.

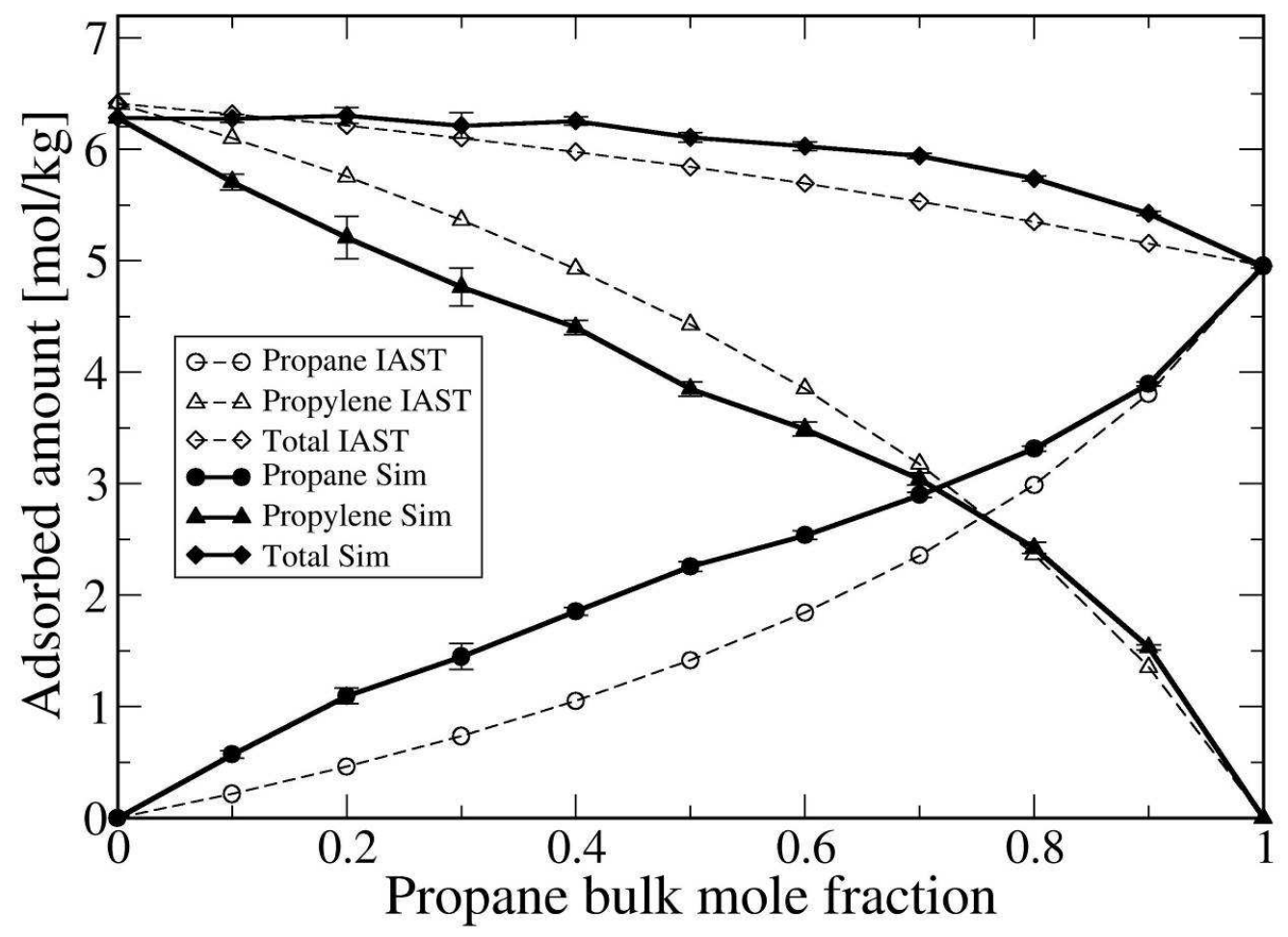

Figure 12 - Binary adsorption isotherms for propane/propylene on CuBTC at $373 \mathrm{~K}$ and $100 \mathrm{kPa}$, with varying molar composition of the bulk mixture. Full lines and solid symbols are predictions from GCMC simulations using the modified potential that accounts for $\mathrm{Cu}-\pi$ interactions, while dashed lines and open symbols are predictions from IAST by fitting experimental pure-component isotherms, as carried out by Jorge et al. [23]. 
Although IAST has been successful in predicting mixure adsorption in several different systems, it is known to fail when the adsorption mechanism moves away from ideality and/or the adsorbent is energetically heterogeneous [58]. This may certainly be the case in this particular system, since propane and propylene adsorb differently on CuBTC. Indeed, a recent study found that IAST fails for several gas mixtures in CuBTC [59]. Thus, the first hypothesis can only be completely ruled out once experimental binary adsorption data for this system become available. The two other hypotheses also cannot be ruled out at present, and it is difficult to distinguish which of the two is most likely. Nevertheless, it is possible that even further improvements in molecular models are necessary (to describe both "standard" LJ interactions and specific $\mathrm{Cu}-\pi$ interactions) before molecular simulation of mixture adsorption in MOFs becomes fully predictive. These aspects are discussed in more detail in the following section.

\subsection{Comparison to previous approaches}

The method developed in this paper is directly comparable to previous work by Jorge and coworkers [22,23] and by Fischer et al. [24,25]. For this purpose, we begin by plotting the potential energy profiles that are actually "felt" by an ethylene molecule when it adsorbs on the OMS, calculated using those two previous approaches and our new method (see Figure 13). Except for the solid circles, which represent the actual DFT profile for ethylene calculated using the protocol described in section 2.2, all other curves were calculated using a Monte Carlo procedure that randomly sampled a very large number of different orientations of the adsorbate for each distance to the $\mathrm{Cu}$ atom, as was done previously to calculate the repulsive contribution (see section 2.3). To obtain the lines shown in Figure 13, the adsorbate-framework interactions included the full LennardJones potential (both repulsion and dispersion) as well as the specific $\mathrm{Cu}-\pi$ attraction as described by each different method. As a consequence, there is a systematic offset between the DFT and the classical profiles, caused by the neglect of dispersion interactions in the DFT calculations. 


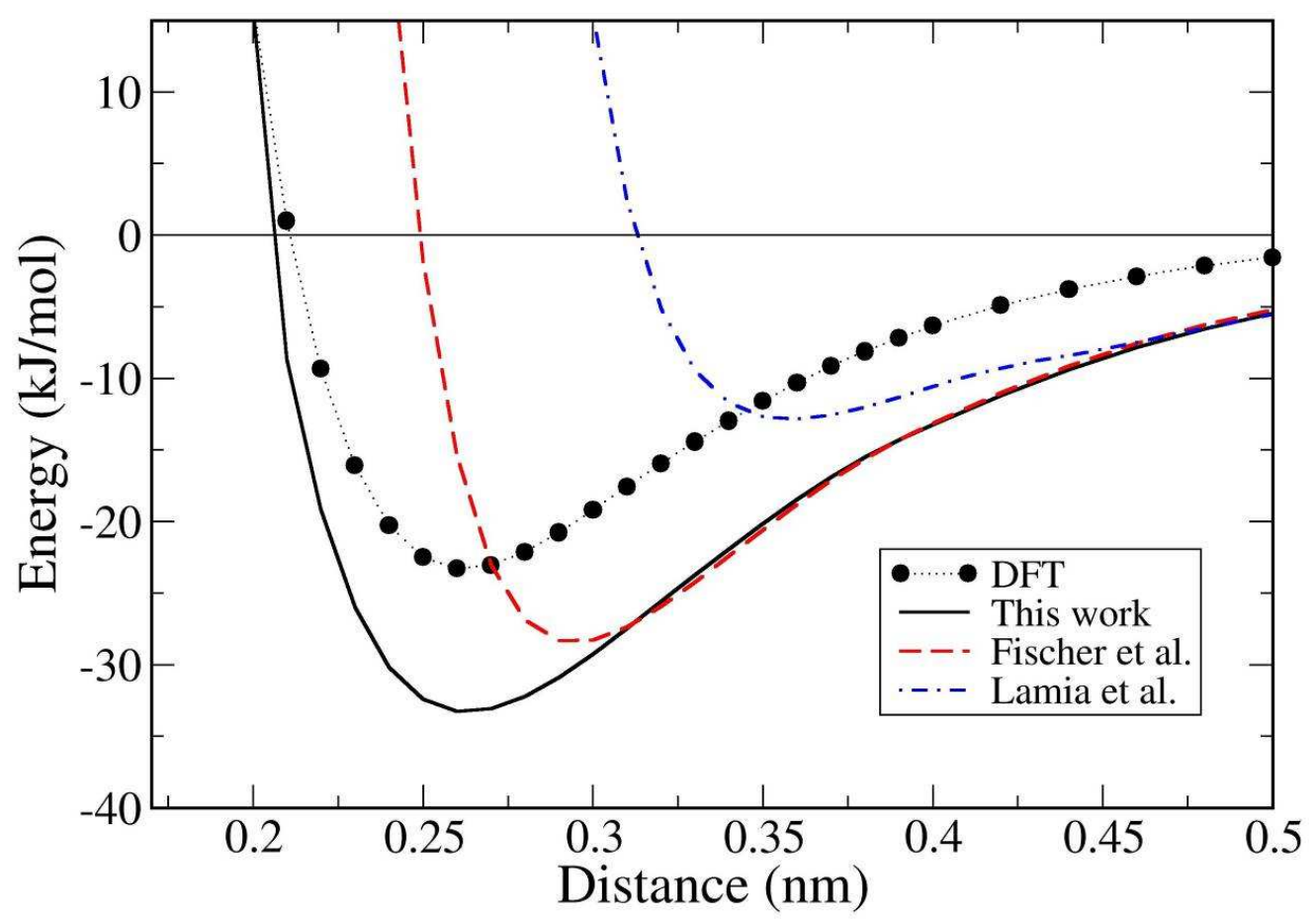

Figure 13 - Potential energy curves obtained in classical Monte Carlo simulations using different approaches for including the $\mathrm{Cu}-\pi$ interactions. The circles represent the DFT energy profile obtained in this work (see Figure 3). The blue dotted-dashed line was obtained from the LJ potential with the adjustments applied by Lamia et al. [22]. The red dashed line was obtained by adding a Morse potential, fitted to reproduce the DFT curve, to the LJ potential, following the approach of Fischer et al. [25]. The full black line was obtained by adding the potential described by equations 4 and 5 to the $\mathrm{LJ}$ potential, using the approach proposed in this paper.

As suggested previously [23], the empirical adjustment of the LJ energy parameter between $\mathrm{Cu}$ and $\mathrm{sp}^{2}$ sites, proposed by Lamia et al. [22] does not yield a physically correct description of the underlying interactions - the potential well is shallower and shifted to significantly longer distances to the metal site. It compensated for this fact by overestimating the interaction energy in the central region of the pore volume, yielding pure-component isotherms that are in good agreement with experiment [22]. However, it is unable to describe binary propane/propylene adsorption [23] because the inherent adsorption mechanism is not correct.

The approach of Fischer et al. [24,25] is more physically reasonable, since it is also based on incorporating results of DFT calculations into GCMC simulations of adsorption. Indeed, the potential energy profile for ethylene on CuBTC obtained using their approach (red dashed line in Figure 13) is much closer to the actual DFT curve (solid circles). However, apart from the expected offset due to neglect of dispersion interactions in DFT, observed at distances above $0.28 \mathrm{~nm}$, there is a significant shift in the potential well to longer distances $(0.295 \mathrm{~nm}$ in the Fischer et al. profile compared to 0.260 $\mathrm{nm}$ in the DFT curve) and a steeper repulsive region starting at much longer distances to the metal site. This effect is caused by a "double-counting" of the repulsive contribution to the adsorption energy - in the approach of Fischer et al., the DFT curve (which includes both the $\mathrm{Cu}-\pi$ interaction and repulsion) is directly fitted to a function and included into the GCMC calculations using the LJ 
potential (which includes both dispersion and repulsion). As such, in their model the adsorbate molecules experience twice the amount of repulsion that they should, leading to the effect observed in Figure 13 and to a significant underestimation of the amount adsorbed at all temperatures and pressures (see Figure 14).

Conversely, our approach adequately accounts for the repulsive contribution to the DFT results, by estimating its magnitude using the WCA approximation (see section 2.3). As a consequence, the potential energy profile for our model only differs from the DFT curve by the expected offset due to dispersion interactions (compare solid circles to black line in Figure 13). More importantly, our simulated adsorption isotherms agree remarkably well with experimental data (Figure 14). Our results highlight the need to properly take into account the different contributions to the adsorption energy in order to accurately model adsorption in MOFs with open metal sites. Indeed, it is possible that the underestimation of the amount adsorbed observed by Fischer et al. for acetylene and carbon dioxide on CuBTC [24] was precisely due to the above-mentioned "double-counting" of repulsion. It would be interesting in the future to apply our new method to those systems to verify this hypothesis.

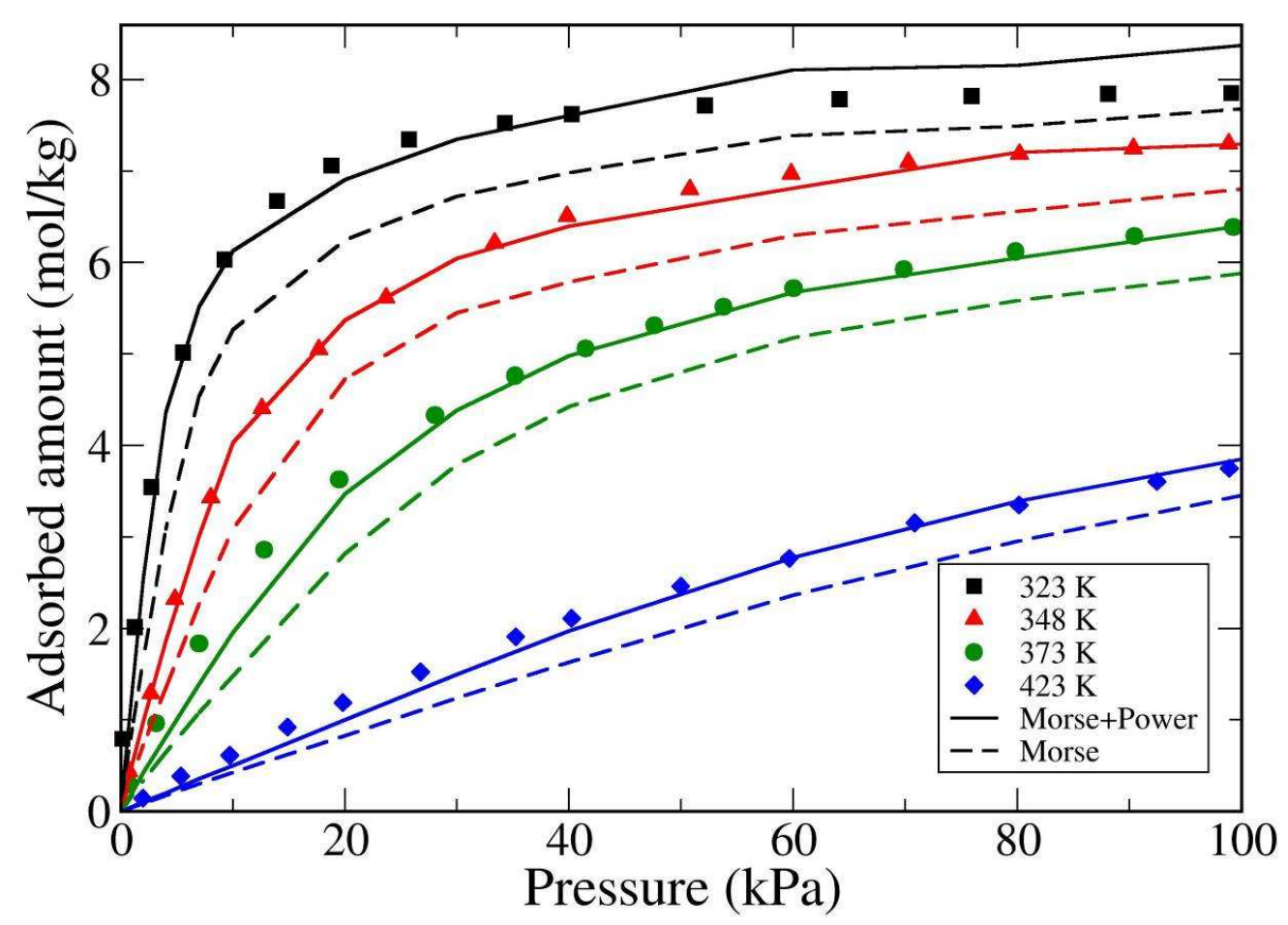

Figure 14 - Comparison between experimental adsorption isotherms (points) and simulation results (lines), for propylene on CuBTC at several temperatures. All simulations included the $\mathrm{Cu}-\pi$ interaction, and were scaled by a factor of 0.84 . The dashed lines were obtained by fitting a Morse potential to the DFT results (approach of Fischer et al. [25]), while the full lines were obtained using our new approach (equations 1-4). Experimental data are from Lamia et al. [22].

Finally, it is instructive to compare our approach to that of Chen et al. [26]. Unfortunately, their method was applied to methane adsorption, and it is not clear how it could be applied to more complex molecules that are not spherically symmetric (such as propylene), thus precluding a 
quantitative comparison. Nevertheless, a qualitative comparison between these two different approaches to the same problem is useful to suggest possible routes for improvement in modelling these complex systems. We begin by stating the assumptions of our method:

1. DFT calculations correctly account for specific $\mathrm{Cu}-\pi$ interactions.

2. DFT results do not take into account any dispersion interactions (i.e., equation 2 is valid).

3. The DREIDING force field correctly accounts for dispersion and repulsion contributions to adsorbate-framework interactions.

4. Non-specific electrostatic interactions between the adsorbate and the framework are either negligible or are implicitly included into the $\mathrm{LJ}$ and $\mathrm{Cu}-\pi$ contributions.

5. The CuBTC sample contains about $16 \%$ of excluded volume for adsorption.

We have no particular reason to doubt the validity of the first assumption, since DFT methods have been widely used with success for describing coordination chemistry problems $[60,61]$. This is reinforced by the success of both our own and previous studies [24,25] in modeling adsorption in MOFs with OMS. As for the second statement, the degree to which it is valid will clearly depend on the details of the DFT functional and basis set. The DFT profile for ethane (Figure 6) suggests that the particular combination used here (PBE with a DNP basis set) may be capturing some of the dispersion interactions, at least close to the framework. If this is the case, then our modelling approach will suffer from a small but systematic error. A possible way to circumvent this problem is to use much higher-level quantum mechanical methods (e.g., coupled-cluster techniques extrapolated to infinite basis set), but at present they are too computationally demanding for application to reasonably sized systems. Chen et al. address this issue by applying a clever method to account for the limitations of DFT - correction factors for individual atoms are determined by comparing DFT with high-level coupled-cluster calculations in small molecules, and these are then used to correct DFT energies on larger fragments, thus accounting, among other things, for missing dispersion interactions [62-64]. We are currently carrying out high-level ab initio studies on these systems in order to determine to what extent dispersion contributions can be described by conventional and dispersion-corrected DFT, and will report these results in a forthcoming publication.

Assumption 3 was tested by comparing simulated and experimental adsorption of propane, for which specific interactions should be practically absent. Good agreement relied on assuming a fixed percentage of excluded volume in the real material (assumption 5), and thus these two assumptions cannot currently be decoupled. Indeed, although the scaling factor was found to be consistently applicable for all temperatures, pressures, adsorbates and compositions, lending support to our approach, we cannot rule out the possibility that a more accurate molecular model than DREIDING may obviate the need for such a rescaling. In this context, it is interesting to notice that Chen et al. found that several commonly used force fields (DREIDING, UFF and OPLS-AA) overestimated methane adsorption in CuBTC at very low temperatures, suggesting an inherent inadequacy of these models for describing even dispersion-dominated interactions with the framework [26]. Clearly, further efforts are necessary to firmly establish the validity of standard force fields for modelling adsorption in MOFs, preferably using as test cases materials that do not possess OMS or framework 
flexibility. This also relies on the ability to synthesise MOF samples with a high degree of purity and on the availability of highly accurate experimental data on such samples, particularly at low pressures and temperatures.

Our model also assumes that non-specific electrostatic interactions are negligible in this system. This is supported by the success of united-atom neutral models in predicting adsorption of alkanes and alkenes in other adsorbents, such as zeolites [36,37,40,41]. Nevertheless, alkenes do possess some degree of polarity, and the use of models that explicitly consider electrostatic interactions may improve the accuracy of the predictions, as suggested recently by GutierrezSevillano et al. [65]. Therefore, an interesting path for future development would involve explicitly including Coulombic interactions into our approach, and testing to what extent they improve the adsorption predictions. It should also be noted that for other adsorbates (e.g., acetylene), electrostatic interactions are likely to play a more significant role, and thus an extension of our method in this direction is essential for general applicability. In this context, it would also be interesting to examine the effect of partially hydrating the CuBTC material on its selectivity for olefin/paraffin separations.

The major advantage of Chen et al.'s approach relative to our own is precisely that it does not rely on a molecular model to accurately describe LJ and Coulombic interactions with the framework. Instead, they assume that their corrected DFT calculations already account for all of these contributions, and use them directly to compute the adsorbate-framework interactions during GCMC [26]. On the other hand, as mentioned previously, it is questionable that the method can be applied to non-spherically symmetric adsorbates, a limitation from which our approach does not suffer. Furthermore, transferability becomes an issue, since the approach of Chen et al. would potentially require extensive DFT calculations to be performed for each adsorbate/framework combination (and perhaps even some high-level coupled-cluster calculations to calibrate their correction factors). Our approach, however, has at least the potential to be transferable to similar adsorbates and similar metal centers, as the work of Fischer et al. [25] demonstrated. Thus, we believe it has greater scope for use as a generally applicable tool to model adsorption in MOFs with OMS.

\section{4 - Conclusions}

In this paper we present a new approach for modeling adsorption in metal-organic frameworks that contain open metal sites, and apply it to propane/propylene separations in CuBTC. To achieve this, we carry out density functional theory calculations to compute the interaction energy profile between the olefin double bond and the $\mathrm{Cu}$ metal site. The repulsive contribution, estimated using a classical Lennard-Jones potential and the Weeks-Chandler-Andersen approximation, is then subtracted from this curve to isolate the contribution due to the specific attractive interaction between the metal and the $\pi$-orbitals of the olefin. The underlying energy profile is fitted to a functional form composed of a Morse potential and a power law term, which is then incorporated into classical-level Grand Canonical Monte Carlo simulations to compute the adsorbate-framework interactions. GCMC simulations using this modified potential are able to successfully predict pure-component adsorption isotherms of propane and propylene at several different temperatures. Significant differences were observed in the adsorption mechanism of these two species, whereby propane adsorbs mainly in the small pores of $\mathrm{CuBTC}$ while propylene also prefers to adsorb close to the metal sites. Mixture 
adsorption predictions from GCMC using the new model are in reasonable agreement with predictions from Ideal Adsorbed Solution Theory, but some important discrepancies were observed. Experimental measurements of binary propane/propylene adsorption in CuBTC would be extremely valuable to determine which of those two approaches is more accurate.

We systematically examined the effect of several variables on the performance of the model. The size of the molecular cluster used to represent the framework in the DFT calculations was shown to have a relatively minor effect. In this context, it was shown that small $\mathrm{Cu}_{2}$ (formate) ${ }_{4}$ clusters may be useful models of these systems when computational time is limited (e.g., in high-level quantum mechanical calculations). The protocol for optimization of the adsorbate-framework complex was also found to have a minor effect, provided excessive deformations of the cluster are prevented.

A major improvement of our work relative to previous approaches is a more consistent consideration of the different contributions to the adsorption energy, allowing one to isolate the contribution from the specific metal-adsorbate interaction. We found this to be crucial for obtaining accurate adsorption predictions, and application of our approach to other systems is likely to lead to similar improvements. Nevertheless, there are several possible avenues to improve our approach. First of all, because it depends on the use of standard force fields to describe the repulsion and dispersion contributions, significant improvements are expected if more accurate models for these interactions in MOFs become available. In fact, our method is itself an important contribution toward this goal, since it allows one to decouple specific interactions with OMS from "standard" dispersion and repulsion interactions. For similar reasons, consideration of models that explicitly include electrostatic interactions by way of point-charges may also improve the accuracy of the results. Finally, the use of more accurate quantum mechanical methods, particularly those that are able to describe dispersion interactions, would be a valuable addition to our method. Efforts in this direction are already underway.

The dependence of our approach on reliable force fields for describing repulsion, dispersion and electrostatic interactions between the adsorbate and the framework is both a blessing and a curse. Alternative approaches that do not rely on such force fields [26] have perhaps a greater potential for producing very accurate results. However, our approach is, at least at present, more amenable for transferability and general application by the simulation community. In any event, we believe these recent efforts constitute major steps forward in our molecular-level understanding of adsorption in MOFs with unsaturated metal centers, and will certainly lead in the near future to the development of an accurate and generally applicable strategy for predicting adsorption in these complex systems.

\section{Supporting Information}

Additional figures (S1-S5) that complement the discussion in the main paper. This material is available free of charge via the Internet at http://pubs.acs.org.

\section{Acknowledgements}


This work is supported by projects PTDC/EQU-EQU/099423/2008, PEst-C/EQB/LA0020/2011 and PEst-C/CTM/LA0011/2011, financed by FEDER through COMPETE - Programa Operacional Factores de Competitividade and by FCT - Fundação para a Ciência e a Tecnologia. Thanks are also due to FCT for Programa Ciência 2007. M. Fi. gratefully acknowledges a postdoctoral fellowship from the German Research Foundation (DFG grant FI 1800/1-1).

\section{References}

[1] Murray, L. J.; Dinca, M.; Long, J. R. Hydrogen storage in metal-organic frameworks. Chem. Soc. Rev. 2009, 38, 1294.

[2] Li, J.; Sculley, J.; Zhou, H. Metal-organic frameworks for separations. Chem. Rev., 2012, 112, 869.

[3] Li, J.; Kuppler, R. J.; Zhou, H. Selective gas adsorption and separation in metal-organic frameworks. Chem. Soc. Rev. 2009, 38, 1477.

[4] Getman, R. B.; Bae, Y.; Wilmer, C. E.; Snurr, R. Q. Review and analysis of molecular simulations of methane, hydrogen, and acetylene storage in metal-organic frameworks. Chem. Rev., 2012, 112, 703.

[5] Lee, J.; Farha, O. K.; Roberts, J.; Scheidt, K. A.; Nguyen, S. T.; Hupp, J. T. Metal-organic framework materials as catalysts. Chem. Soc. Rev. 2009, 38, 1450.

[6] Yoon, M.; Srirambalaji, R.: Kim, K. Homochiral metal-organic frameworks for asymmetric heterogeneous catalysis. Chem. Rev., 2012, 112, 1196.

[7] Allendorf, M. D.; Bauer, C. A.; Bhakta, R. K.; Houk, R. J. Luminescent metal-organic frameworks. Chem. Soc. Rev. 2009, 38, 1330.

[8] Kreno, L. E.; Leong, K.; Farha, O. K.; Allendorf, M.; Van Duyne, R. P.; Hupp, J. T. Metal-organic framework materials as chemical sensors. Chem. Rev., 2012, 112, 1105.

[9] Zhou, H.; Long, J. R.; Yaghi, O. M. Introduction to metal-organic frameworks. Chem. Rev., 2012, 112,673 .

[10] Ferey, G.; Mellot-Draznieks, C.; Serre, C.; Millange, F.; Dutour, J.; Surble, S.; Margiolaki, I. A chromium terephthalate-based solid with unusually large pore volumes and surface area. Science, 2005, 309, 2040.

[11] Bourrelly, S.; Llewellyn, P. L.; Serre, C.; Millange, F.; Loiseau, T.; Ferey, G. Different adsorption behaviors of methane and carbon dioxide in the isotypic nanoporous metal terephthalates MIL-53 and MIL-47. J. Am. Chem. Soc., 2005, 127, 13519.

[12] Wu, H.; Zhou, W.; Yildirim, T. High-capacity methane storage in metal-organic frameworks M2(dhtp): the important role of open metal sites. J. Am. Chem. Soc., 2009, 131, 4995. 
[13] Mu, B.; Li, F.; Walton, K. S. A novel metal-organic coordination polymer for selective adsorption of $\mathrm{CO}_{2}$ over $\mathrm{CH}_{4}$. Chem. Commun. 2009, 18, 2493.

[14] Rowsell, J. L. C.; Yaghi, O. M. Metal-organic frameworks: a new class of porous materials. Microporous Mesoporous Mater., 2004, 73, 3.

[15] Smit, B.; Maesen, T. L. M. Molecular simulations of zeolites: adsorption, diffusion, and shape selectivity. Chem. Rev., 2008, 108, 4125.

[16] Jorge, M.; Seaton, N.A. Predicting adsorption of water/organic mixtures using molecular simulation. AIChE J., 2003, 49, 2059.

[17] Yun, J. H.; Düren, T.; Keil, F. J.; Seaton, N. A. Adsorption of methane, ethane, and their binary mixtures on MCM-41: an experimental evaluation of methods for the prediction of adsorption equilibrium. Langmuir, 2002, 18, 2693.

[18] Düren, T.; Sarkisov, L.; Yaghi, O. M.; Snurr, R. Q. Design of new materials for methane storage. Langmuir, 2004, 20, 2683.

[19] Keskin, S.; Liu, J.; Rankin, R. B.; Johnson, J. K.; Sholl, D.S. Progress, opportunities, and challenges for applying atomically detailed modeling to molecular adsorption and transport in metal-organic framework materials. Ind. Eng. Chem. Res. 2009, 48, 2355.

[20] Neimark, A. V.; Coudert, F. X.; Boutin, A.; Fuchs, A. H. Stress-based model for the breathing of metal-organic frameworks. J. Phys. Chem. Lett., 2010, 1, 445.

[21] Tafipolsky, M.; Amirjalayer, S.; Schmid, R. Ab initio parametrized MM3 force field for the metal-organic framework MOF-5. J. Comput. Chem., 2007, 28, 1169.

[22] Lamia, N.; Jorge, M.; Granato, M. A.; Almeida Paz, F. A.; Chevreau, H.; Rodrigues, A. E. Adsorption of propane, propylene and isobutane on a metal-organic framework: Molecular simulation and experiment. Chem. Eng. Sci. 2009, 64, 3246.

[23] Jorge, M.; Lamia, N.; Rodrigues, A. E. Molecular simulation of propane/propylene separation on the metal-organic framework CuBTC. Colloids Surf., A 2010, 357, 27.

[24] Fischer, M.; Hoffmann, F.; Fröba, M. New microporous materials for acetylene storage and $\mathrm{C}_{2} \mathrm{H}_{2} / \mathrm{CO}_{2}$ separation: Insights from molecular simulations. ChemPhysChem. 2010, 11, 2220.

[25] Fischer, M.; Kuchta, B.; Firlej, L.; Hoffmann, F.; Fröba, M. Accurate prediction of hydrogen adsorption in metal-organic frameworks with unsaturated metal sites via a combined densityfunctional theory and molecular mechanics approach. J. Phys. Chem. C 2010, 114, 19116.

[26] Chen, L.; Grajciar, L.; Nachtigall, P.; Düren, T. Accurate prediction of methane adsorption in a metal-organic framework with unsaturated metal sites by direct implementation of an ab initio derived potential energy surface in GCMC simulation. J. Phys. Chem. C 2011, 115, 23074.

[27] Yang, R. T. Adsorbents: Fundamentals and Applications, Wiley, Hoboken, 2003. 
[28] Bao, Z.; Alnemrat, S.; Yu, L.; Vasiliev, I.; Ren, Q.; Lu, X.; Deng, S. Adsorption of ethane, ethylene, propane, and propylene on a magnesium-based metal-organic framework. Langmuir, 2011, 27,13554 .

[29] Bae, Y.; Lee, C. Y.; Kim, K. C.; Farha, O. K.; Nickias, P.; Hupp, J. T.; Nguyen, S. T.; Snurr, R. Q. High propene/propane selectivity in isostructural metal-organic frameworks with high densities of open metal sites. Angew. Chem. Int. Ed. 2012, 51, 1857.

[30] Chui, S. S. Y.; Lo, S. M. F.; Charmant, J. P. H.; Orpen, A. G.; Williams, I. D. A chemically functionalizable nanoporous material [Cu3(TMA)2(H2O)3]n. Science 1999, 283, 1148.

[31] Norman, G. E.; Filinov, V. S., Investigations of phase transitions by a Monte-Carlo method. High Temperature 1969, 7, 216.

[32] Peng, D.; Robinson, D. B. A new two-constant equation of state. Ind. Eng. Chem. Fundamen. 1976, 15, 59 .

[33] Myers, A. L.; Monson, P. A. Adsorption in porous materials at high pressure: Theory and experiment. Langmuir 2002, 18, 10261.

[34] Mayo, S. L.; Olafson, B. D.; Goddard III, W. A. DREIDING: a generic force field for molecular simulations. J. Phys. Chem. 1990, 94, 8897.

[35] Rappé, A. K.; Casewit, C. J.; Colwell, K. S.; Goddard III, W. A.; Skiff, W. M. UFF, a full periodic table force field for molecular mechanics and molecular dynamics simulations. J. Am. Chem. Soc. 1992, 114, 10024.

[36] Dubbeldam, D.; Calero, S.; Vlugt, T. J. H.; Krishna, R.; Maesen, T. L. M.; Smit, B. United atom force field for alkanes in nanoporous materials. J. Phys. Chem. B 2004, 108, 12301.

[37] Jakobtorweihen, S.; Hansen, N.; Keil, F. Molecular simulation of alkene adsorption in zeolites. Mol. Phys. 2005, 103, 471.

[38] Martin, M. G.; Siepmann, J. I. Transferable potentials for phase equilibria. 1. United-atom description of n-alkanes. J. Phys. Chem. B 1998, 102, 2569.

[39] Wick, C. D.; Martin, M. G.; Siepmann, J. I. Transferable potentials for phase equilibria. 4. United-atom description of linear and branched alkenes and alkylbenzenes. J. Phys. Chem. B 2000, 104, 8008 .

[40] Granato, M. A.; Vlugt, T. J. H.; Rodrigues, A. E. Molecular simulation of propane-propylene binary adsorption equilibrium in zeolite 13X. Ind. Eng. Chem. Res. 2007, 46, 7239.

[41] Granato, M. A.; Lamia, N.; Vlugt, T. J. H.; Rodrigues, A. E. Adsorption equilibrium of isobutane and 1-butene in zeolite 13X by molecular simulation. Ind. Eng. Chem. Res. 2008, 47, 6166.

[42] Delley, B. An all-electron numerical method for solving the local density functional for polyatomic molecules. J. Chem. Phys. 1990, 92, 508. 
[43] Delley, B. From molecules to solids with the DMol ${ }^{3}$ approach. J. Chem. Phys. 2000, 113, 7756.

[44] Materials Studio Version 5.5; Accelrys Inc.: San Diego, 2011.

[45] Perdew, J. P.; Burke, K.; Ernzerhof, M. Generalized gradient approximation made simple. Phys. Rev. Lett. 1996, 77, 3865.

[46] Watanabe, T.; Sholl, D. S. Molecular chemisorption on open metal sites in $\mathrm{Cu}_{3}$ (benzenetricarboxylate) $)_{2}$ : A spatially periodic density functional theory study. J. Chem. Phys. 2010, 133, 094509.

[47] Dion, M.; Rydberg, H.; Schroder, E.; Langreth, D. C.; Lundqvist, B. I. Van der Waals density functional for general geometries. Phys. Rev. Lett. 2004, 92, 246401.

[48] Johnson, E. R.; Mackie, I. D.; DiLabio, G. A. Dispersion interactions in density-functional theory. J. Phys. Org. Chem. 2009, 22, 1127.

[49] Grimme, S. Semiempirical GGA-type density functional constructed with a long-range dispersion correction. J. Comput. Chem., 2006, 27, 1787.

[50] Iikura, H.; Tsuneda, T.; Yanai, T.; Hirao, K. A long-range correction scheme for generalizedgradient-approximation exchange functionals. J. Chem. Phys. 2001, 115, 3540.

[51] Chai, J. -D.; Head-Gordon, M. Long-range corrected hybrid density functionals with damped atom-atom dispersion corrections. Phys. Chem. Chem. Phys. 2008, 10, 6615.

[52] Sato, T.; Nakai, H. Local response dispersion method. II. Generalized multicenter interactions. $J$. Chem. Phys. 2010, 133, 194101.

[53] Weeks, J. D.; Chandler, D.; Andersen, H. C. Role of repulsive forces in determining the equilibrium structure of simple liquids. J. Chem. Phys. 1977 54, 5237.

[54] Roszak, S.; Balasubramanian, K. Reaction of the copper dimer with ethylene. A theoretical study. Chem. Phys. Lett. 1994, 231, 18.

[55] Fischer, M.; Hoffmann, F.; Fröba, M. Molecular simulation of hydrogen adsorption in metalorganic frameworks. Colloids Surf., A 2010, 357, 35.

[56] Grajciar, L., Bludský, O., Nachtigall, P. Water adsorption on coordinatively unsaturated sites in CuBTC MOF. J. Phys. Chem. Lett. 2010, 23, 3354.

[57] Myers, A. L.; Prausnitz, J. M. Thermodynamics of mixed-gas adsorption. AIChE J. 1965, 11, 121.

[58] Murthi, M.; Snurr, R. Q. Effects of molecular siting and adsorbent heterogeneity on the ideality of adsorption equilibria. Langmuir, 2004, 20, 2489.

[59] Cessford, N. F., Seaton, N. A., Düren, T. Evaluation of ideal adsorbed solution theory as a tool for the design of metal-organic framework materials. Ind. Eng. Chem. Res. 2012, 51, 4911 
[60] Neese, F. Prediction of molecular properties and molecular spectroscopy with density functional theory: From fundamental theory to exchange-coupling. Coord. Chem. Rev. 2009, 253, 526.

[61] Cramer, C. J.; Truhlar, D. G. Density functional theory for transition metals and transition metal chemistry. Phys. Chem. Chem. Phys. 2009, 11, 10757.

[62] Bludský, O.; Rubeš, M.; Soldan, P.; Nachtigall, P. Investigation of the benzene-dimer potential energy surface: DFT/CCSD(T) correction scheme. J. Chem. Phys. 2008, 128, 114102.

[63] Rubeš, M.; Kysilka, J.; Nachtigall, P.; Bludský, DFT/CC investigation of physical adsorption on a graphite (0001) surface. O. Phys. Chem. Chem. Phys. 2010, 12, 6438.

[64] Zukal, A.; Pulido, A.; Gil, B.; Nachtigall, P.; Bludský, O.; Rubeš, M.; Čejka, J. Experimental and theoretical determination of adsorption heats of $\mathrm{CO}_{2}$ over alkali metal exchanged ferrierites with different Si/Al ratio. Phys. Chem. Chem. Phys. 2010, 12, 6413.

[65] Gutierrez-Sevillano, J. J.; Caro-Perez, A.; Dubbeldam, D.; Calero, S. Analysis of the ITQ-12 zeolite performance in propane-propylene separations using a combination of experiments and molecular simulations. J. Phys. Chem. C 2010, 114, 14907. 
Table of Contents Graphic

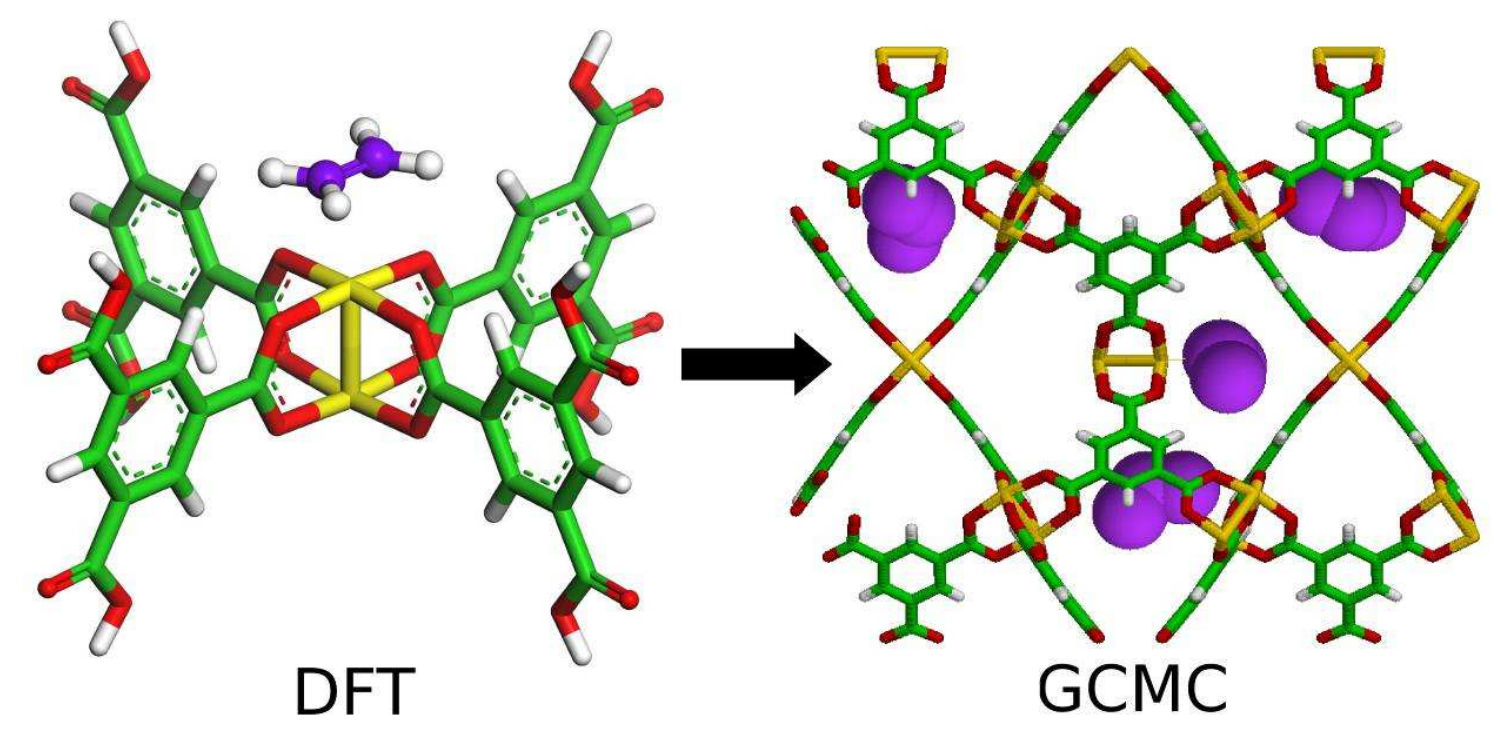

